
tumorigenesis genes in non-small-cell lung cancer

\title{
Li Peng
}

Wuhan University Zhongnan Hospital

Yuwei Liu

Wuhan University Zhongnan Hospital

Jing Chen

Wuhan University Zhongnan Hospital

Xuebei Du

Wuhan University Zhongnan Hospital

Renwei Zhang

Wuhan University Zhongnan Hospital

Mengxin Cheng

Wuhan University Zhongnan Hospital

Ying Wu

Wuhan University Zhongnan Hospital

Min Chen

Wuhan University Zhongnan Hospital

\section{Ya Zhong}

Wuhan University Zhongnan Hospital

\section{Dan Shen}

Wuhan University Zhongnan Hospital

\section{Ling Chen}

Wuhan University Zhongnan Hospital

Xujun Ye ( $\nabla$ wdxjy@whu.edu.cn)

Wuhan University Zhongnan Hospital

\section{Research article}

Keywords: APEX1, RNA-seq, Alternative Splicing, Non-Small-Cell Lung Cancer (NSCLC)

Posted Date: November 16th, 2020

DOI: https://doi.org/10.21203/rs.3.rs-108181/v1 
License: (c) (i) This work is licensed under a Creative Commons Attribution 4.0 International License. Read Full License 


\section{Abstract}

Background: Aberrant alternative splicing (AS) contributes to tumor progression. Previous studies have shown that apurinic-apyrimidinic endonuclease-1 (APEX1) is involved in tumor progression. It is unknown whether APEX1 functions in tumor progression by regulation of AS. It is also unknown whether APEX1 can regulate non-small-cell lung cancer (NSCLC) proliferation and apoptosis.

Methods: We analyzed APEX1 expression levels in 517 lung NSCLC samples from the TCGA (Cancer Genome Atlas) database. The impact of APEX1 over expression on A549 cell proliferation and apoptosis was detected by the methyl thiazolyl tetrazolium assay and by flow cytometry. The transcriptome of A549 cells with and without APEX1 over expression was determined by Illumina sequencing, followed by analysis of AS. RT-qPCR validated APEX1 in A549 cells.

Results: We have successfully applied RNA-seq technology to demonstrate APEX1 regulation of AS. APEX1 expression was shown to be upregulated in NSCLC samples and to reduce cell proliferation and induce apoptosis of A549 cells. Further, APEX1 regulated AS of key tumorigenesis genes involved in cancer proliferation and apoptosis within the MAPK and Wnt signaling pathways. Each of these pathways are involved in lung cancer progression. Validated AS events regulated by APEX1 were located in key tumorigenesis genes; AXIN1 (axis inhibition protein 1), GCNT2 (N-acetyl glucosaminyl transferase 2), and SMAD3 (SMAD Family Member 3). These genes encode signaling pathway transcription regulatory factors.

Conclusions: We found that increased expression of APEX1 in NSCLC is an independent prognostic factor related to tumor progression. Therefore, APEX1 regulation of AS may serve as a molecular marker or therapeutic target for NSCLC treatment.

\section{Introduction}

With increasing worldwide incidence and mortality, lung cancer has become the major cause of cancer death and a serious clinical issue [1, 2]. Non-small-cell lung cancer (NSCLC) is the most prevalent and heterogeneous subtype of lung cancer comprised of adenocarcinoma, squamous cell carcinoma, and large cell carcinoma [3]. Improved patient prognosis and survival requires improvements in screening for early lung cancer biomarkers, development of therapeutic efficacy predictors, and new treatment drugs [4]. These improvements would greatly enhance patient outcomes for this malignant disease.

Alternative splicing (AS) is a major means by which protein diversity is generated [5]. Data from genomewide studies suggest that more than $90 \%$ of human genes undergo AS [6]. It has become apparent that coordinated splicing networks regulate tissue and organ development, and that AS is important to physiological function and to human developmental processes [7].

Evidence suggests that AS contributes to cell differentiation, lineage determination, tissue-identity acquisition, tissue maintenance, and organ development [7]. Systematic and coordinated AS alterations 
of functionally linked RNA-binding proteins (RBPs) could impact carcinogenesis [8]. Hence, AS as a model of cancer, may provide new insights into cancer treatment targets. Furthermore, AS and the RBPs which control this process are often deregulated in cancer [9].

RBPs are important regulators of AS and have important roles: in cancer progression, they are key components of RNA metabolism and regulate the temporal, spatial and functional dynamics of RNAs. Altering the expression of RBPs has profound implications for cellular physiology by affecting RNA processes from pre-mRNA splicing to protein translation. Recent genetic and proteomic studies of animal models demonstrated RBPs to be involved in many human diseases ranging from neurologic disorders to cancer [10]. RBPs are known modulators of cell growth and proliferation; thus, their dysfunction is likely to have cancer implications. Furthermore, using mass spectrometry, more than 1000 RBPs have been identified in eukaryotic cells, many of which regulate splicing [11]. Thus, exploration of RBP regulatory mechanisms associated with AS will deepen our understanding of cancer.

Altered expression of apurinic-apyrimidinic endonuclease-1 (APEX1) APEX1, known as a RBP, is often observed in human tumors [12]. Accumulating evidence indicates that aberrant APEX1 expression is a general phenomenon of human cancer [13]. Further, APEX1 has been shown to play an important role in the progression of lung cancer, as well as genome stability maintenance [14]. Elevated and ectopic expression of APEX1 in tumor tissue is closely linked to a poor prognosis for lung cancer $[12,15]$.

Although previous studies have suggested involvement of APEX1 in tumor progression, it remains unclear whether APEX1 regulates AS in NSCLC. The aim of this study is to determine whether APEX1 indirectly regulates proliferation and apoptosis of NSCLC through AS. To accomplish this aim, we assessed the relationships among APEX1, cellular proliferation, apoptosis regulation, and cancer progression through specific signaling pathways. The approach was to analyze APEX1 expression levels in 517 lung NSCLC samples from the TCGA (Cancer Genome Atlas) database and as well, the impact of APEX1 on A549 cell proliferation and apoptosis. Transcriptomes of A549 cells with and without APEX1 overexpression were evaluated by Illumina sequencing, followed by analysis of AS regulation. Reverse transcriptionquantitative polymerase chain reaction (RT-qPCR) was performed to validate APEX1 in A549 cells and clinical NSCLC samples. We found that APEX1 expression was upregulated in NSCLC samples, and that APEX1 overexpression led to reduced cell proliferation and apoptosis. Further, APEX1-regulated AS of many genes, which are related to cancer proliferation and apoptosis pathways, the Wnt signaling pathway and the MAPK signaling pathway. Taken together, these results provide major insight into APEX1-regulated AS and the development of NSCLC. Further analysis of APEX1-regulated AS will focus on a precise understanding of the signaling networks that direct tumorigenesis and which may serve as potential APEX1-targeted therapies. In summary, these findings provide mechanistic insight into the means by which AS contributes to NSCLC tumorigenesis and may also serve as the basis for identification of relevant cancer treatment biomarkers.

\section{Materials And Methods}




\section{Cloning and plasmid construction}

The primer pairs used for thermal fusion were designed using CE Design V1.04. Each primer contains a gene-specific fragment and a 17-30 bp sequence of pIRES-hrGFP-1a vector.

F-primer: agcccgggeggatccgaattcATGCCGAAGCGTGGGAAA.

R-primer: gtcatccttgtagtcctcgagCAGTGCTAGGTATAGGGTGATAGG.

The pIRES-hrGFP-1a vector was digested with EcoRI and Xhol (NEB, Ipswich, MA, US) at $37^{\circ} \mathrm{C}$ for $2 \mathrm{~h} \sim 3 \mathrm{~h}$. The enzyme-digested vector was then electrophoresed in a 1.0\% agarose gel and purified with a Qiagen column kit. Total RNA was isolated from A549 cells using Trizol (Life Technology, Carlsbad, CA, USA). The purified RNA was transcribed by oligo dT primers to obtain CDNA. The synthetic insert was then amplified by PCR. The linearized vector was digested with EcoRI and Xhol (NEB, Ipswich, MA, USA) and the PCR insert were added to PCR microtubes to connect with a ClonExpress ${ }^{\circledR}$ II One Step Cloning Kit (Vazyme, Nanjing, China). Plasmids were introduced into Escherichia coli by chemical transformation. The cells were seeded onto LB agar plates containing $1 \mu \mathrm{L} / \mathrm{mL}$ ampicillin and incubated overnight at $37^{\circ} \mathrm{C}$. Colonies were screened by colony PCR ( 28 cycles) using universal primers (located on the backbone vector). The inserted sequence was verified by Sanger sequencing.

\section{Cell culture and transfections}

NSCLC A549 cells (Procell Life Science \& Technology Co., Ltd., Wuhan, China) were cultured in Dulbecco's Modified Eagle Medium (DMEM) at $37^{\circ} \mathrm{C}$, containing $10 \%$ fetal bovine serum (FBS), $100 \mu \mathrm{g} / \mathrm{mL}$ streptomycin, and $100 \mathrm{U} / \mathrm{mL}$ penicillin in a 5\% CO2 incubator. Cell growth was observed with a light microscope, with digestion and passage performed when the cell confluence reached $80 \%$ or more. According to the manufacturer's protocol, Lipofectamine 2000 (Invitrogen, Carlsbad, CA, USA) was used to transfect A549 cells with a plasmid overexpressing APEX1 and an empty plasmid. The transfected cells were harvested after $48 \mathrm{~h}$ for RT-qPCR analysis.

\section{RNA extraction and sequencing}

Before extracting RNA, A549 cells were ground to a fine powder. Total RNA was extracted with Trizol (Life Technology, Carlsbad, CA, USA). The RNA was further purified with two phenol-chloroform treatments and then digested with RQ1 DNase (Promega, Madison, WI, USA) to remove DNA. The quality and quantity of purified RNA was measured at $260 \mathrm{~nm} / 280 \mathrm{~nm}$ (A260/A280) using a Smartspec Plus (BioRad, Sacramento, CA, USA). The integrity of RNA was further verified by $1.5 \%$ agarose gel electrophoresis. For each sample, $1 \mu \mathrm{g}$ of total RNA was used for RNA-seq library preparation with a VAHTS Stranded mRNAseq Library Prep Kit (Vazyme, Nanjing, China). The polyadenylated mRNAs were purified and fragmented, and then converted into double-stranded cDNA. After end repair and A tailing steps, the DNA was ligated to the VAHTS RNA adaptor (Vazyme, Nanjing, China). The purified ligation product corresponding to 200500 bps was digested with heat-labile UDG, and the single-stranded cDNA was amplified, purified, 
quantified, and stored at $-80^{\circ} \mathrm{C}$ before sequencing. For high-throughput sequencing, the library was prepared according to the manufacturer's instructions and analyzed with the Illumina HiSeq X Ten system for $150 \mathrm{nt}$ paired-end sequencing.

\section{Assessment of APEX1 overexpression}

GAPDH (glyceraldehyde-3-phosphate dehydrogenase) was used as a control gene to assess the effects of APEX1 overexpression. cDNA synthesis was completed by standard procedures and RT-qPCR was performed with a Bio-Rad S1000 with Bestar SYBR Green RT-PCR master mix (DBI Bioscience, Shanghai, China). Primer information is provided in Attachment 1. The 2- $\Delta \Delta C T$ method was then used to normalize the concentration of each transcript to GAPDH mRNA levels [16]. With GraphPad Prism software (version TM, GraphPad software, San Diego, CA, USA), paired Student $t$ tests were performed.

\section{RNA-Seq raw data cleaning and alignment}

Original reads containing more than 2-N bases were discarded. Then adaptors and low-quality bases were trimmed from raw sequencing reads using the FASTX-Toolkit (Version 0.0.13). Short reads less than 16 nt were deleted. After that, tophat2 [17] aligned the clean reads with the GRch38 genome, allowing four mismatches. Uniquely mapped reads were used for gene read counts and FPKM calculations (fragments per kilo base of transcripts per million fragments mapped).

\section{Differentially expressed genes (DEGs) analysis}

The R Bioconductor package edgeR [18] was utilized to identify the differentially expressed genes (DEGs). A false discovery rate of $<0.05$ and fold change $>2$ or $<0.5$ were the cut-off criteria for identifying DEGs.

\section{Alternative splicing analysis}

Alternative splicing events (ASEs) and regulated alternative splicing events (RASEs) between the samples were defined and quantified using the $A B L$ pipeline[19]. In short, the detection of $A B L$, as ten types of ASEs, is based on splice junction reads, including exon skipping (ES), alternative 5 ' splice site (A5SS), alternative 3'splice site (A3SS), intron retention (IR), mutually exclusive exons (MXE), mutually exclusive 5'UTRs (5pMXE), mutually exclusive 3'UTRs (3pMXE), cassette exon, A3SS\&ES, and A5SS\&ES. In order to evaluate the ASE regulated by RNA-binding proteins, a Student's t-test was performed to assess the importance of ratio alteration of AS events. The events that were significant at the cut-off $p$ value (corresponding to the cut-off of the $5 \%$ false discovery rate) were considered ASE for RBP control.

\section{Reverse transcription qPCR validation of DEGs and AS events}

To validate the RNA-seq data, qRT-PCR was performed on some DEGs. Primer information is found in Additional file 1. Total RNA remaining from RNA-seq library preparation was used for RT-qPCR. M-MLV reverse transcriptase (Vazyme, Nanjing, China) was used to reverse transcribe RNA to cDNA. According to the manufacturer's instructions, real-time PCR was performed by SYBR Green PCR Reagents Kit (Yeasen, 
Shanghai, China). PCR amplifications were performed in triplicate for each sample. The RNA expression levels of all the genes were normalized relative to GAPDH. qRT-PCR assays were also performed for ASE validation. To detect alternative isoforms, we used crossover primers. This sequence covers the connection of constitutive exons and the relative primers in constitutive exons. Design boundaryspanning primers for alternative exons according to "model exons" was used to detect model splicing, or "altered exons" to detect altered splicing.

\section{Methyl thiazolyl tetrazolium (MTT) assay}

A549 cells were dissociated enzymatically, quantified, and then incubated in a 96-well plate (3000 cells/well) for $24 \mathrm{~h}$. After treatment, $20 \mu \mathrm{L}$ of MTT solution (Sigma-Aldrich, St. Louis, MO, USA, $5 \mathrm{mg} / \mathrm{mL}$ ) was added to each well and incubated for another $4 \mathrm{~h}$. Then, the supernatant was discarded carefully and dimethyl sulfoxide (DMSO, Sigma-Aldrich, St. Louis, MO, USA) added to each well and incubated for 10 min, followed by slight shaking for $15 \mathrm{~min}$. Absorbance at $490 \mathrm{~nm}$ was assessed with a microplate reader (Molecular Devices, Sunnyvale, CA, USA). All processing groups were repeated at least three times.

\section{Flow cytometry}

More than $1 \times 10^{5}$ cells were harvested and analyzed using a FACS Calibur flow cytometer (Becton Dickinson, Canton, MA, USA). Apoptotic cells were identified using flow cytometry with FITC-Annexin V Staining Detection Kits in accordance with manufacturer's instruction. Numerical values were analyzed with CELL Quest 3.0 software (BD, Canton, MA, USA). Results were averages of three independent experiments.

\section{Western blot analysis}

A549 cells were obtained from each experimental treatment group and solubilized on ice for $30 \mathrm{~min}$ in radio immunoprecipitation assay (RIPA) buffer. The protein was stored at $4^{\circ} \mathrm{C}$ after the protein concentration was determined using a BCA Protein Assay kit (Thermo Scientific, Rockford, IL, USA). Fifteen $\mu \mathrm{g}$ of protein for each group were boiled for 10 min with protein denaturants, separated by $10 \%$ sodium dodecyl sulfate polyacrylamide gel electrophoresis (SDS-PAGE), and transferred to PVDF membranes (Millipore, Billerica, MA, USA). The membranes were blocked with $5 \%$ skim milk diluted in phosphate-buffered saline containing $0.1 \%$ Tween-20 (PBST) for $1 \mathrm{~h}$ at room time. The membranes were incubated at $4^{\circ} \mathrm{C}$ overnight with primary antibodies. After washing with PBST three times (5 min each), the membranes were incubated with horseradish peroxidase (HRP)-conjugated antibodies for $45 \mathrm{~min}$. An ECL analysis system (Santa Cruz, Piscataway, NJ, USA) was used for detection following the manufacture's protocol. Western blot quantification was performed using Image Processing and Analysis software.

\section{Functional enrichment analysis}


To assess the functional categories of DEGs, Gene Ontology (GO) terms and Kyoto Encyclopedia of Genes and Genomes (KEGG) pathways were identified with a KOBAS 2.0 server [20]. The Hypergeometric test and the Benjamini-Hochberg FDR controlling procedure were used to define enrichment terms.

\section{Downloading RNA-seq data for NSCLC samples}

RNA-seq data for NSCLC samples were downloaded from the Cancer Genome Atlas (TCGA) database for analysis of APEX 1 expression and regulation of AS in NSCLC. The data presented in this publication are available using GEO Series accession number GSE146875.

\section{Results}

\section{APEX1 expression is upregulated during all NSCLC stages}

The TCGA database is an open-access tumor database that provides detailed and comprehensive genomic datasets for download and analysis. In order to investigate APEX1 differential expression and clinical relevance for all NSCLC stages, we used the clinical and transcriptomic data from the TCGA database (https://cancergenome.nih.gov/), which included 517 NSCLC and 59 normal samples. We found APEX1 to be significantly upregulated in 517 NSCLC samples compared to 59 normal tissue samples (Log-rank test, $p<0.01$, Fig. 1A and Additional file 2). The 517 NSCLC samples were classified into different stages, including stage I (5), IA (132), IB (140), IIA (50), IIB (71), IIIA (73), IIIB (11), and IV (26) (Fig. 1B). Collectively, these data demonstrate APEX1 to be upregulated and its levels to be correlated with malignant features of human NSCLCs.

\section{Overexpression of APEX1 induces apoptosis and inhibits proliferation of A549 cells.}

To analyze the effect of APEX1 on cell proliferation and apoptosis, A549 cells were transfected with control and APEX1 overexpression vectors (Fig. 2A. Primer sequence information is shown in Materials and Methods). As shown in Fig. 2A and 2D, over expression of APEX1 in A549 cells was confirmed by RTqPCR and western blot analysis. As presented in Fig. 2B, cell proliferation was significantly reduced by APEX1 overexpression when compared with control $(p<0.05)$, suggesting that APEX1 overexpression inhibits cell proliferation. Conversely, overexpression of APEX1 increased apoptosis of A549 cells $(p<$ 0.05 , Fig. $2 \mathrm{C}$ ) as judged by staining with two fluorescent dyes, Annexin V/FITC and PI, and detection by flow cytometry. Over expression of APEX1 resulted in apoptosis of $14.5 \%$ of A549 cells whereas only $2.74 \%$ of control A549 cells were apoptotic (Fig. 2E, $p<0.05$ ). These results demonstrated that overexpression of APEX1 promoted apoptosis in the NSCLC cell line, A549. The molecular basis for these effects requires further investigation at the molecular level.

\section{Transcriptome Analysis Of Apex1-mediated AS}

The successful overexpression of APEX1 was demonstrated by analyzing the FPKM (fragment per kilobase per million reads) values as detailed in the Materials and Methods (Fig. 3A). In order to gain insight into the role of APEX1 in AS regulation, we performed transcriptome sequencing of A549 cells 
with or without APEX1 overexpression. A total of $81 \mathrm{M} \pm 1.06 \mathrm{M}$ uniquely mapped reads were obtained from APEX1-OE (Overexpression) and control groups, in which approximately 49.75\% 52.92\% splice events were junction reads (details can be found in Additional file 3). These data allowed detection of $72.58 \%$ of annotated exons ( 266,614 out of 367,321 annotated exons), and 179,608 annotated and 254,897 novel splice junctions using Tophat2. To compare the gene expression patterns across individuals, expression values in units of FPKM were calculated using an in-house pipeline. Effective depletion of APEX1 was confirmed in parallel by RNA-seq analysis (Fig. 3A). We further explored APEX1regulated AS events with the RNA-seq dataset using ABLas software [21]. We detected 36,086 known ASEs and 55,088 novel ASEs, excluding intron retention (IR) (details can be found in Additional file 4). By applying a stringent cutoff of a $p$-value $\leq 0.05$ and a changed AS ratio $\geq 0.2$ (See Materials and Methods), we identified 611 high-confidence APEX1-RASEs. Complete RASEs can be found in Additional file 5 and Fig. 3B, including 109 known IR RASEs and 502 non-IR (NIR) RASEs. The majority of RASEs were within alternative 5' splice sites (A5SS, 142 events), alternative 3'splice sites (A3SS, 122 events), exon skipping (ES, 88 events), and cassette exons (CE, 53 events). The other event types included mutually exclusive 5'UTR (5pMXE, 35), mutually exclusive 3'UTR (3pMXE, 9), mutually exclusive exon (MXE, 25), alternative 5' splice site \& exon skipping (A5SS \& ES, 17), and alternative 3' splice site \& exon skipping (A3SS \& ES, 11). These data suggest that APEX1 globally regulates AS events in A549 cells.

A total of 611 genes harbored APEX1-regualted ASEs, termed RASGs. To rule out the possibility that APEX1-regulated AS events could be simply attributed to the APEX1-mediated transcriptional regulation, we analyzed genes whose transcription levels were regulated by APEX1-OE by running edgeR package, which resulted in 86 of such DEGs. As shown in Fig. 3C, only one RASG overlapped with a DEG. To assess the potential biological function of APEX1-regulated AS, RASGs were subjected to GO and KEGG analysis. The top ten $\mathrm{GO}$ biological terms were; activation of cysteine-type endopeptidase activity involved in apoptosis, regulation of transcription, and G2/M transition of mitotic cell cycle (Fig. 3D). Enriched KEGG pathways ( $p \geq 0.05$ ) included; MAPK signaling pathway, Wnt signaling pathway, and carbon metabolism signaling pathway (Fig. 3E). Genes involved in each GO term or KEGG pathway are found in Additional file 6.

\section{Identification of APEX1-regulated AS of cancer-related genes in A549 cells}

To validate APEX1-RASEs identified by RNA-seq, 21 RASEs classified into IR, ES, A5SS, and A3SS were selected for q-PCR validation. Most of these genes in RASEs belonged to enriched functional terms or KEGG pathways. Out of 21 tested events, three alternative splicing events validated by q-PCR agreed with the RNA-seq results. The three validated splicing events were in the following genes, AXIN1 (axis inhibition protein 1), GCNT2 (N-acetyl glucosaminyl transferase 2), and SMAD3 (SMAD Family Member 3). AS of CTBP2 (C-Terminal Binding Protein 2), PPARD (peroxisome proliferator-activated receptor), and FBXW11 (F-Box and WD Repeat Domain Containing 11) were inversely regulated by APEX1 (Fig. 4. and Additional file 7). In summary, these results demonstrate confidence in the APEX1-RASEs and AS data.

\section{Validation of APEX1-regulated gene expression and alternative splicing in NSCLC clinical samples}


In order to validate confidence in the DEGs and ASEs detected in this study, we performed additional experiments with NSCLC clinical samples from the TCGA Lung study. We selected 40 cancer samples with 20 high and 20 low APEX1 expression levels (Fig. 5A). A total of $125 \mathrm{M} \pm 49 \mathrm{M}$ clean reads per sample were downloaded from the TCGA database. Among these, $108 \mathrm{M} \pm 42 \mathrm{M}$ reads per sample uniquely aligned to the human genome, in which junction reads accounted for 14.14-25.10\% (details can be found in Additional file 8). We then analyzed ASEs from the RNA-seq dataset and detected 36,086 known ASEs and 93,066 novel ASEs, without counting intron retention events. Published studies have validated the efficacy of ABas software for detection of ASEs from multiple pairs of samples [21, 22].

By applying a stringent cutoff of $p \leq 0.05$, with changed AS ratio $\geq 0.2$, we identified 4,626 highconfidence RASEs that were associated with APEX1 expression levels in these 40 clinical samples (Fig. 5B). These data suggest that APEX1 extensively regulates ASEs in NSCLC. Genes harboring APEX1RASEs were highly enriched in: apoptotic signaling pathway, cellular protein metabolic process, and G2/M transition of mitotic cell cycle (GO biological process terms, Fig. 5C). Meantime Enriched KEGG pathways $(p \geq 0.05)$ included; cell cycle, ubiquitin mediated proteolysis, and p53 signaling pathway (Fig. 5D). Taken together, these results indicate that APEX1-RASEs may play a role in NSCLC genesis. Because cancer tissues are complicated by multiple cell types and deregulated genes, these potential APEX1-RASEs could contribute to oncogenesis. Further, APEX1 could globally regulate ASEs in NSCLC. To illustrate the consistency of AS between clinical samples and the A549 cell line, we carefully analyzed the expression levels of APEX1-RASs in NSCLC and A549 cells. We found overlap of the RASEs (Fig. 6), with validation by RNA-seq and q-PCR. $p$-value: 3.638768 e-146

\section{Discussion}

APEX1, known as a RBP closely associated with NSCLC, may play a critical role in cancer [23, 24]. However, the involvement of APEX1 in cancer progression, especially its impact on AS of genes within functional signaling pathways, has not been fully elucidated. The main finding of this study is that APEX1 is upregulated in human NSCLC tissues, and that APEX1 increases cellular apoptosis and decreases cellular proliferation by regulation of AS in A549 cells. Further, APEX1 was shown to affect key tumorigenesis genes of the Wnt signaling pathway and the MAPK signaling pathway by regulating AS. These genes include AXIN1, GCNT2, and SMAD3. These results were confirmed in NSCLC tissues from the TAGA database and as well in A549 cells. We believe our results demonstrate, for the first time in lung cancer, the effects of APEX1 upon AS-related genes of the Wnt signaling and MAPK signaling pathways.

Recent evidence has demonstrated APEX1 upregulation in various cancers such as NSCLC and colorectal cancer $[25,26]$. However, the clinical significance of APEX1 in cancer remains unclear, although APEX1 has been shown to be a positive regulator, contributing to the aggressive behavior of colon cancer behaviors [27]. Many studies have suggested increased APEX1 levels to be diagnostic and prognostic for cancer, and that APEX1 may be a therapeutic target for treatment of advanced cancer [28]. APEX1 expression has been inversely correlated with cellular senescence in human primary cells, while in cancer, low levels of APEX1 expression have been associated with increased senescence [29]. Results of this 
study support the hypothesis that APEX1 is cancer-promoting and that AS of cancer-related genes has a critical impact on lung cancer cell biology [30]. Further, the effect of APEX1 on lung cancer cell proliferation and apoptotic pathway-related genes is due to AS. The specific mechanism by which APEX1 induces such effects requires further investigation.

Given the widespread AS perturbations in cancer, it is important to determine how AS events mediate cancer progress [31]. Abundant evidence has shown AS to be associated with lung cancer [32]. Further, evidence has demonstrated that AS alterations in cancer can be caused by changes in expression, amplification, and deletion of RBPs [31,33]. A change in AS events can occur in cancer related genes, which correlate with an increase in cell proliferation and metastasis, the basis for $90 \%$ of all human cancer mortality [34]. It has been established that, in some cases, a relationship exists between a splicing event and an increase in cancer cell proliferation and invasion $[34,35]$. As such, AS may be a hallmark of cancer [36]. By analysis of the GO biological process and KEGG functional pathways in A549 cells and with confirmation in clinical NSCLC samples, AS and APEX1 were found to play critical roles in the regulation of signaling pathways that control cell proliferation and tumorigenesis, including the MAPK and Wnt signaling pathways.

Previous studies found the MAPK signaling pathway to be critically involved in many important cellular processes including protein biosynthesis, cell cycle control, apoptosis, and differentiation [37]. Once activated, the MAPK signaling pathway exerts major effects on cell physiology. Given the many important roles for MAPK pathway signaling, its activation is tightly regulated [38]. The activity of the MAPK signaling pathway is critical to proliferation of normal and cancerous cells. Oncogenic mutations that drive the development of NSCLC often activate this signaling pathway [39]. Sandra et al. found that activation of the MAPK pathway may be beneficial to the survival, proliferation, and spread of lung cells in response to multiple stimuli, with evidence demonstrating high levels of MAPK pathway activity to be detrimental to lung tumors by interference with the neuroendocrine system [39]. In NSCLC patients, other studies found that resulting chimeric proteins functioned to promote cancer cell proliferation, invasion, and anti-apoptosis through activation of MAPK signaling [40]. Herein, we found that greater expression of APEX1 was regulated by AS of genes related to the MAPK signaling pathway, which may be detrimental to NSCLC. The mechanistic basis could be APEX1 regulation of AS alterations that stimulate MAPK signaling pathway components, including genes encoding kinases and transcription factors.

The Wnt signaling pathway is a main regulator of development within the animal kingdom. Dysregulation of the Wnt signaling pathway results in multiple growth-related pathologies and cancer. Further, the Wnt signaling pathway has been shown to be involved in cancer progression, differentiation, migration, genetic stability, and apoptosis [41]. Constituents of Wnt signaling pathway can be either positive or negative, wherein the negative constituents principally act to suppress tumorigenesis, which for cancer can be mutation or loss of function [42]. AS related transcripts of RBPs have been detected in NSCLC and breast tumor tissues and may contribute to altered Wnt signaling [43]. Lung cancer "stemness" can be maintained by targeting negative regulators of the Wnt signaling pathway for degradation, thereby increasing Wnt activity [44]. The mechanism may be, after abnormal activation of Wnt signaling, 
expression of downstream target genes accelerates cell proliferation, producing abnormal proteins and accelerating tumor formation [45]. Collectively, these results suggest AS to be related to RBPs changes in NSCLC, impacting cell signaling in a manner that likely contributes to tumorigenesis [43]. Our findings indicate that suppression of the Wnt signaling pathway, by targeting AS by APEX1, could inhibit NSCLC proliferation and promote apoptosis. Our results further support the concept that targeting differential AS related to APEX1 may be a means by which to reduce cell proliferation and induce apoptosis of tumors in patients with lung cancer. Further investigations are needed to elucidate the regulatory mechanisms by which AS impacts genes related to the MAPK and Wnt signal pathways. These investigations could provide for the development of new means by which to target malignant tumors.

Previous studies have suggested a relationship between AS and RBPs in cancer, which would change expression, amplification, and deletion of specific signaling pathway genes [46]. Based on this study, AS events regulated by APEX1 were mostly located in genes encoding transcription regulation factors in the MAPK and Wnt signaling pathways. By qPCR and RNA-seq we identified several ASEs of cancer related genes that were regulated by APEX1 including; AXIN1, GCNT2, SMAD3, CTBP2, PPARD, and FBXW11 (Fig. 6). Further, compared to the control group, the APEX1 related genes were upregulated and consistent with APEX1-RASEs identified by ABLas RNA-seq and qPCR. Further, several APEX1-RASEs were involved in genes encoding protein kinases such as; AXIN1, GCNT2, SMAD3, CTBP2, PPARD, and FBXW11, which play important roles in different signaling pathways or cellular processes. Mechanistically, we have demonstrated APEX1 inhibition to induce a rapid downregulation of AXIN1. AXIN1 is an important regulator of beta-catenin, which is frequently mutated in human hepatocellular carcinoma. Transduction of wild-type (AXIN1) induces apoptosis of colon cancer cells [47]. AXIN1 has been found in various human cancers, including those of the colon, liver, endometrium, ovary, prostate, and stomach [48]. Previously, reduced expression of AXIN1 was related to poor differentiation of lung cancer. Therefore, AXIN1 may provide a new target for therapeutic intervention in lung cancer. In this study, we found GCNT2 to be overexpressed in highly metastatic NSCLC samples. Functional studies showed that ectopic expression of GCNT2 enhanced cell detachment, adhesion to endothelial cells, cell migration and invasion in vitro, and lung metastasis of breast cancer cells in vivo [49]. So GCNT2 may be a novel gene contributing to metastasis with preferential expression in lung cancer, implying that blocking the TGF $\beta /$ GCNT2 signal pathway is a promising approach for targeting metastatic NSCLC. Furthermore, we found that APEX1 can activate the TGF $\beta /$ SAMD3 signal pathway by promoting lung cancer cells proliferation, as described previously [50]. For the TGF $\beta /$ SMAD3 signaling pathway, receptor-activated SAMD complexes activate or repress their target gene promoters. SMAD cofactors are a group of SMADbinding proteins that promote recruitment of SMAD complexes to promoters [51]. The mechanistic basis could be that APEX1 overexpression affects specific signaling pathways such as MAPK and Wnt, which are very important to cancer progression, differentiation, and apoptosis by up-regulating SMAD3 expression.

These results confirm that APEX1 can indirectly regulate development of NSCLC by affecting AS of the above-mentioned genes, thereby controlling proliferation and apoptosis-related signaling pathways. In point of fact, RBPs are overexpressed in a wide variety of cancers and the silencing of these genes 
induces apoptosis in cancer cells but not in normal cells [52]. AS of cancer-related genes may have a critical impact on lung cancer cell biology. Considering that splicing machinery is changed in lung cancer, it is important to keep in mind that many other AS found in lung cancer tissues may be "passengers" rather than "drivers" of malignant transformation [32]. Previous studies have found that alterations in genes encoding RBPs are pervasive in cancer, characterize different tumor types, and can explain many of the AS changes observed in lung cancer. We confirm that APEX1 plays a very important role in the biology of NSCLC by regulating AS of key tumorigenesis genes related to proliferation and apoptosis signaling pathways. Taken together, our results indicate that APEX1 may play a role in promoting cancer by inducing the expression of many oncogenes, although identification of the precise mechanism requires further study.

\section{Conclusions}

In this study, we have successfully applied RNA-seq technology to demonstrate APEX1 regulation of AS, which is consistent with its reported role as a signaling adaptor, a kinase, and a mRNA-associated protein. We demonstrated APEX1 expression to be up-regulated in NSCLC samples, and that APEX1 over expression may reduce cell proliferation and induce apoptosis. In addition, we confirm that APEX1 regulates the AS of key tumorigenesis genes involved in cancer proliferation and apoptosis pathways, the MAPK signaling pathway and the Wnt signaling pathway, which mediate lung cancer progression. Therefore, APEX1 regulation of AS may serve as a molecular marker or therapeutic target for NSCLC treatment. However, functional studies will be required to confirm the biologic relevance of APEX1associated AS in lung cancer.

\section{Abbreviations}

A3SS

Alternative 3'splice site; A5SS:Alternative 5'splice site; APEX1:apurinic-apyrimidinic endonuclease-1; AS:Aberrant alternative splicing; ASEs:Alternative splicing events; AXIN1:axis inhibition protein 1; CE:Cassette exon; CTBP2:C-Terminal Binding Protein 2; DEGs:Differentially expressed genes; DMEM:Dulbecco's Modified Eagle Medium; DMSO:dimethyl sulfoxide; ES:Exon skipping; FBS:fetal bovine serum; FBXW11:F-Box and WD Repeat Domain Containing 11; FPKM:Fragments per kilo base of exon model per million fragments mapped; GAPDH:glyceraldehyde-3-phosphate dehydrogenase; GCNT2:Nacetyl glucosaminyl transferase 2; GO:Gene Ontology; HRP:horseradish peroxidase; IR:intron retention; KEGG:Kyoto Encyclopedia of Genes and Genomes; MEX:Mutually exclusive exons; 5pMXE:mutually exclusive 5'UTRs; 3pMXE:mutually exclusive 3'UTRs; NSCLC:non-small-cell lung cancer; MTT:Methyl thiazolyl tetrazolium; OE:Overexpression; PPARD:peroxisome proliferator-activated receptor; RASEs:Regulated alternative splicing events; RBPs:RNA-binding proteins; RIPA:radio immunoprecipitation assay; RT-qPCR:Quantitative reverse transcription polymerase chain reaction; SDS-PAGE:sodium dodecyl sulfate polyacrylamide gel electrophoresis; SMAD3:SMAD Family Member 3; TCGA:The Cancer Genome Atlas 


\section{Declarations}

\section{Ethics approval and consent to participate}

Not applicable, A549 cell line was purchased from CCTCC (China Center for Type Culture Collection, Wuhan, Hubei, China), and did not require ethics approval for their use in this study.

\section{Consent for publication}

Not applicable.

\section{Conflict of Interest Statement}

The authors declare that they have no competing interests.

\section{Funding}

This work was supported by the Zhongnan Hospital of Wuhan University Science, Technology, and Innovation Seed Fund (Grant No. znpy2019084), and the Fundamental Research Funds for the Central Universities (Grant No. 2042019kf0138).

\section{Authors' contributions}

L.P., Y.L., J.C., X.D. and X.Y. contributed to the study design. R.Z., M.C., Y.W., M.C., Y.Z., D.S. performed the experiment and/or performed data analysis. L.P., L.C. and X.Y. prepared the manuscript. All authors read and approved the final manuscript.

\section{Acknowledgments}

We thank International Science Editing (http://www.internationalscienceediting.com) for editing this manuscript.

\section{References}

1. Boumahdi S, de Sauvage FJ. The great escape: tumour cell plasticity in resistance to targeted therapy. Nature reviews Drug discovery. 2020;19(1):39-56.

2. Siegel RL, Miller KD, Jemal A. Cancer statistics, 2019. Cancer J Clin. 2019;69(1):7-34.

3. Sanchez-Cespedes M. Lung cancer biology: a genetic and genomic perspective. Clinical translational oncology: official publication of the Federation of Spanish Oncology Societies of the National Cancer Institute of Mexico. 2009;11(5):263-9. 
4. Konopa K. Do we have markers to select patients for adjuvant therapies of non-small-cell lung cancer? Annals of oncology: official journal of the European Society for Medical Oncology. 2010;21(Suppl 7):vii199-202.

5. Coomer AO, Black F, Greystoke A, Munkley J, Elliott DJ. Alternative splicing in lung cancer. Biochimica et biophysica acta Gene regulatory mechanisms. 2019;1862(11-12):194388.

6. Wang ET, Sandberg R, Luo S, Khrebtukova I, Zhang L, Mayr C, Kingsmore SF, Schroth GP, Burge CB. Alternative isoform regulation in human tissue transcriptomes. Nature. 2008;456(7221):470-6.

7. Baralle FE, Giudice J. Alternative splicing as a regulator of development and tissue identity. Nature reviews Molecular cell biology. 2017;18(7):437-51.

8. Xiong Y, Deng Y, Wang K, Zhou H, Zheng X, Si L, Fu Z. Profiles of alternative splicing in colorectal cancer and their clinical significance: A study based on large-scale sequencing data. EBioMedicine. 2018;36:183-95.

9. Venables JP, Klinck R, Koh C, Gervais-Bird J, Bramard A, Inkel L, Durand M, Couture S, Froehlich U, Lapointe $\mathrm{E}$, et al. Cancer-associated regulation of alternative splicing. Nat Struct Mol Biol. 2009;16(6):670-6.

10. Lukong KE, Chang KW, Khandjian EW, Richard S. RNA-binding proteins in human genetic disease. Trends Genet. 2008;24(8):416-25.

11. Song X, Zeng Z, Wei H, Wang Z. Alternative splicing in cancers: From aberrant regulation to new therapeutics. Semin Cell Dev Biol. 2018;75:13-22.

12. Wang D, Xiang DB, Yang XQ, Chen LS, Li MX, Zhong ZY, Zhang YS. APE1 overexpression is associated with cisplatin resistance in non-small cell lung cancer and targeted inhibition of APE1 enhances the activity of cisplatin in A549 cells. Lung cancer. 2009;66(3):298-304.

13. Noike T, Miwa S, Soeda J, Kobayashi A, Miyagawa S. Increased expression of thioredoxin-1, vascular endothelial growth factor, and redox factor- 1 is associated with poor prognosis in patients with liver metastasis from colorectal cancer. Human pathology. 2008;39(2):201-8.

14. Mohibi S, Chen X, Zhang J. Cancer the'RBP'eutics-RNA-binding proteins as therapeutic targets for cancer. Pharmacol Ther. 2019;203:107390.

15. Yoo DG, Song YJ, Cho EJ, Lee SK, Park JB, Yu JH, Lim SP, Kim JM, Jeon BH. Alteration of APE1/ref-1 expression in non-small cell lung cancer: the implications of impaired extracellular superoxide dismutase and catalase antioxidant systems. Lung cancer. 2008;60(2):277-84.

16. Livak KJ, Schmittgen TD. Analysis of relative gene expression data using real-time quantitative PCR and the 2(-Delta Delta C(T)) Method. Methods. 2001;25(4):402-8.

17. Kim D, Pertea G, Trapnell C, Pimentel H, Kelley R, Salzberg SL. TopHat2: accurate alignment of transcriptomes in the presence of insertions, deletions and gene fusions. Genome biology. 2013;14(4):R36.

18. Robinson MD, McCarthy DJ, Smyth GK. edgeR: a Bioconductor package for differential expression analysis of digital gene expression data. Bioinformatics. 2010;26(1):139-40. 
19. Yao Y, Deng Q, Sun C, Song W, Liu H, Zhou Y. A genome-wide analysis of the gene expression profiles and alternative splicing events during the hypoxia-regulated osteogenic differentiation of human cartilage endplate-derived stem cells. Mol Med Rep. 2017;16(2):1991-2001.

20. Xie C, Mao X, Huang J, Ding Y, Wu J, Dong S, Kong L, Gao G, Li CY, Wei L. KOBAS 2.0: a web server for annotation and identification of enriched pathways and diseases. Nucleic acids research 2011, 39(Web Server issue):W316-322.

21. Xia H, Chen D, Wu Q, Wu G, Zhou Y, Zhang Y, Zhang L. CELF1 preferentially binds to exon-intron boundary and regulates alternative splicing in HeLa cells. Biochimica et biophysica acta Gene regulatory mechanisms. 2017;1860(9):911-21.

22. Jin L, Li G, Yu D, Huang W, Cheng C, Liao S, Wu Q, Zhang Y. Transcriptome analysis reveals the complexity of alternative splicing regulation in the fungus Verticillium dahliae. BMC Genomics. 2017;18(1):130.

23. Abbotts R, Madhusudan S. Human AP endonuclease 1 (APE1): from mechanistic insights to druggable target in cancer. Cancer treatment reviews. 2010;36(5):425-35.

24. Craveiro Sarmento AS, Gomes Lima J, de Souza Timoteo AR, Galvao Ururahy MA, Antunes de Araujo A, Carvalho Vasconcelos R, Candido Dantas VK, Fassarella Agnez-Lima L. Araujo de Melo Campos $\mathrm{JT}$ : Changes in redox and endoplasmic reticulum homeostasis are related to congenital generalized lipodystrophy type 2. Biochimica et biophysica acta Molecular cell biology of lipids. 2020;1865(4):158610.

25. Wang $L$, Chen R, Zhang Y. miR-296-3p targets APEX1 to suppress cell migration and invasion of nonsmall-cell lung cancer. Oncology letters. 2019;18(3):2612-8.

26. Kim HB, Lim HJ, Lee HJ, Park JH, Park SG. Evaluation and Clinical Significance of Jagged-1activated Notch Signaling by APEX1 in Colorectal Cancer. Anticancer research. 2019;39(11):6097105.

27. Kim MH, Kim HB, Yoon SP, Lim SC, Cha MJ, Jeon YJ, Park SG, Chang IY, You HJ. Colon cancer progression is driven by APEX1-mediated upregulation of Jagged. The Journal of clinical investigation 2013.

28. Tummanatsakun D, Proungvitaya T, Roytrakul S, Limpaiboon T, Wongkham S, Wongkham C, Silsirivanit A, Somintara O, Sangkhamanon S, Proungvitaya S. Serum Apurinic/Apyrimidinic Endodeoxyribonuclease 1 (APEX1) Level as a Potential Biomarker of Cholangiocarcinoma. Biomolecules. 2019;26(9):413.

29. Li M, Yang X, Lu X, Dai N, Zhang S, Cheng Y, Zhang L, Yang Y, Liu Y, Yang Z, et al. APE1 deficiency promotes cellular senescence and premature aging features. Nucleic acids research. 2018;46(11):5664-77.

30. Ben-Dov C, Hartmann B, Lundgren J, Valcarcel J. Genome-wide analysis of alternative pre-mRNA splicing. J Biol Chem. 2008;283(3):1229-33.

31. Li Y, Sahni N, Pancsa R, McGrail DJ, Xu J, Hua X, Coulombe-Huntington J, Ryan M, Tychhon B, Sudhakar D, et al. Revealing the Determinants of Widespread Alternative Splicing Perturbation in 
Cancer. Cell reports. 2017;21(3):798-812.

32. Pio R, Montuenga LM. Alternative splicing in lung cancer. Journal of thoracic oncology: official publication of the International Association for the Study of Lung Cancer. 2009;4(6):674-8.

33. Sveen A, Kilpinen S, Ruusulehto A, Lothe RA, Skotheim RI. Aberrant RNA splicing in cancer; expression changes and driver mutations of splicing factor genes. Oncogene. 2016;35(19):2413-27.

34. Hagen RM, Ladomery MR. Role of splice variants in the metastatic progression of prostate cancer. Biochemical Society transactions. 2012;40(4):870-4.

35. Oltean S, Bates DO. Hallmarks of alternative splicing in cancer. Oncogene. 2014;33(46):5311-8.

36. Ladomery M. Aberrant alternative splicing is another hallmark of cancer. International journal of cell biology. 2013;2013:463786.

37. Keshet Y, Seger R. The MAP kinase signaling cascades: a system of hundreds of components regulates a diverse array of physiological functions. Methods in molecular biology. 2010;661:3-38.

38. Yaeger R, Corcoran RB. Targeting Alterations in the RAF-MEK Pathway. Cancer discovery. 2019;9(3):329-41.

39. Cristea S, Sage J. Is the Canonical RAF/MEK/ERK Signaling Pathway a Therapeutic Target in SCLC? Journal of thoracic oncology: official publication of the International Association for the Study of Lung Cancer. 2016;11(8):1233-41.

40. Wicki A, Mandala M, Massi D, Taverna D, Tang H, Hemmings BA, Xue G. Acquired Resistance to Clinical Cancer Therapy: A Twist in Physiological Signaling. Physiological reviews. 2016;96(3):80529.

41. Chen P, Shi P, Du G, Zhang Z, Liu L. Wnt/beta-Catenin, Carbohydrate Metabolism, and PI3K-Akt Signaling Pathway-Related Genes as Potential Cancer Predictors. Journal of healthcare engineering. 2019;2019:9724589.

42. Polakis P. Drugging Wnt signalling in cancer. EMBO J. 2012;31(12):2737-46.

43. Misquitta-Ali CM, Cheng E, O'Hanlon D, Liu N, McGlade CJ, Tsao MS, Blencowe BJ. Global profiling and molecular characterization of alternative splicing events misregulated in lung cancer. Molecular cellular biology. 2011;31(1):138-50.

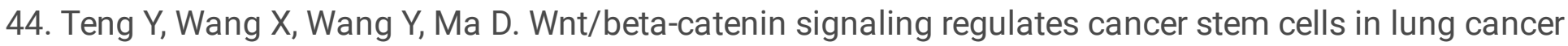
A549 cells. Biochem Biophys Res Commun. 2010;392(3):373-9.

45. Ghosh N, Hossain U, Mandal A, Sil PC. The Wnt signaling pathway: a potential therapeutic target against cancer. Ann N Y Acad Sci. 2019;1443(1):54-74.

46. Fu XD, Ares M Jr. Context-dependent control of alternative splicing by RNA-binding proteins. Nature reviews Genetics. 2014;15(10):689-701.

47. Ishiguro H, Tsunoda T, Tanaka T, Fujii Y, Nakamura Y, Furukawa Y. Identification of AXUD1, a novel human gene induced by AXIN1 and its reduced expression in human carcinomas of the lung, liver, colon and kidney. Oncogene. 2001;20(36):5062-6. 
48. Sunaga N, Kohno T, Kolligs FT, Fearon ER, Saito R, Yokota J. Constitutive activation of the Wnt signaling pathway by CTNNB1 (beta-catenin) mutations in a subset of human lung adenocarcinoma. Genes Chromosom Cancer. 2001;30(3):316-21.

49. Zhang H, Meng F, Wu S, Kreike B, Sethi S, Chen W, Miller FR, Wu G. Engagement of I-branching \{beta\}-1, 6-N-acetylglucosaminyltransferase 2 in breast cancer metastasis and TGF-\{beta\} signaling. Cancer research. 2011;71(14):4846-56.

50. Ciaramella V, Sasso FC, Di Liello R, Corte CMD, Barra G, Viscardi G, Esposito G, Sparano F, Troiani T, Martinelli E, et al. Activity and molecular targets of pioglitazone via blockade of proliferation, invasiveness and bioenergetics in human NSCLC. Journal of experimental clinical cancer research: CR. 2019;38(1):178.

51. Sakai S, Ohhata T, Kitagawa K, Uchida C, Aoshima T, Niida H, Suzuki T, Inoue Y, Miyazawa K, Kitagawa M. Long Noncoding RNA ELIT-1 Acts as a Smad3 Cofactor to Facilitate TGFbeta/Smad Signaling and Promote Epithelial-Mesenchymal Transition. Cancer research. 2019;79(11):2821-38.

52. Martinez-Montiel N, Rosas-Murrieta NH, Anaya Ruiz M, Monjaraz-Guzman E, Martinez-Contreras R. Alternative Splicing as a Target for Cancer Treatment. International journal of molecular sciences $2018,19(2)$.

\section{Figures}

A

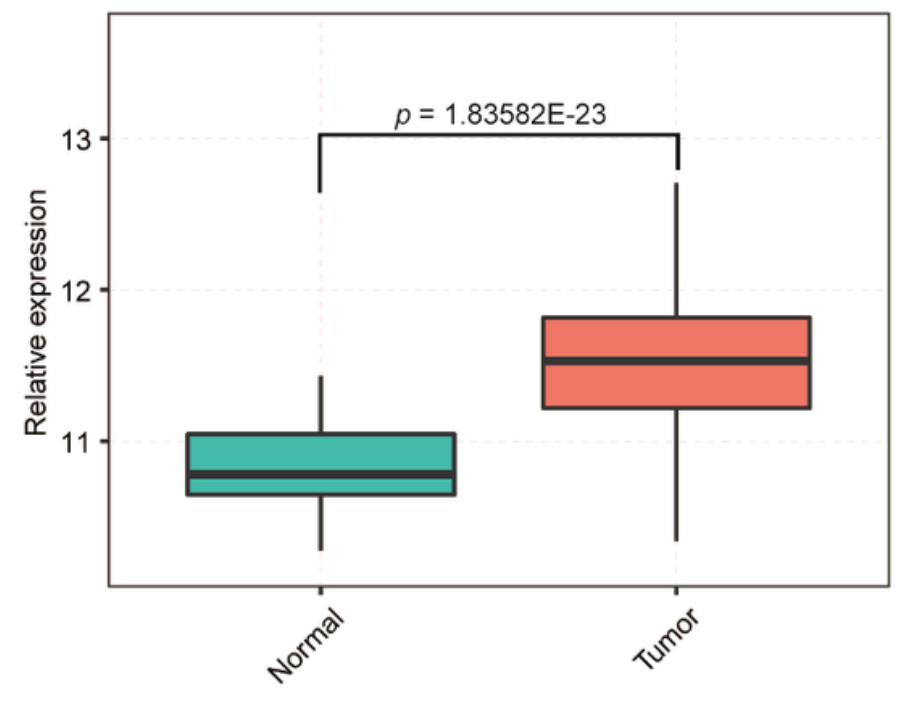

B

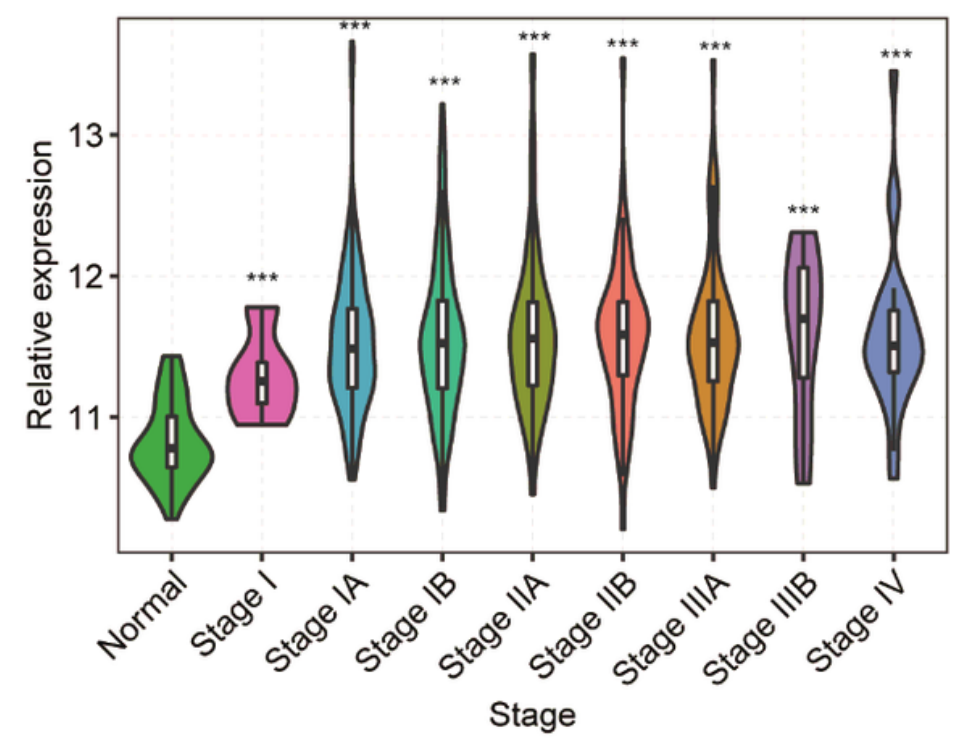

Figure 1

APEX1 expression in NSCLC samples based on the TCGA database. (A) Column chart of APEX1 expression in 517 NSCLC samples and 59 normal samples. APEX1 was significantly higher in lung cancer tissues compared with normal tissue samples. (B) APEX1 distribution of expression levels during 
different stages of NSCLC samples. NSCLC was staged based on the standards of the International Association for Lung Cancer Research (IASLC) stage (Log-rank test, $p<0.05$ ).

A

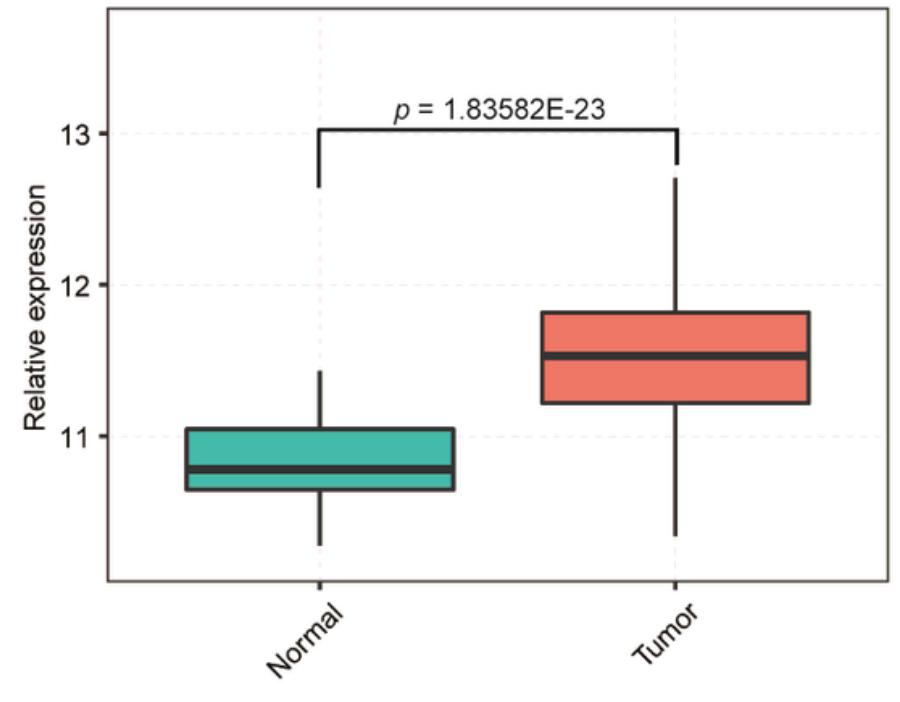

$\mathrm{B}$

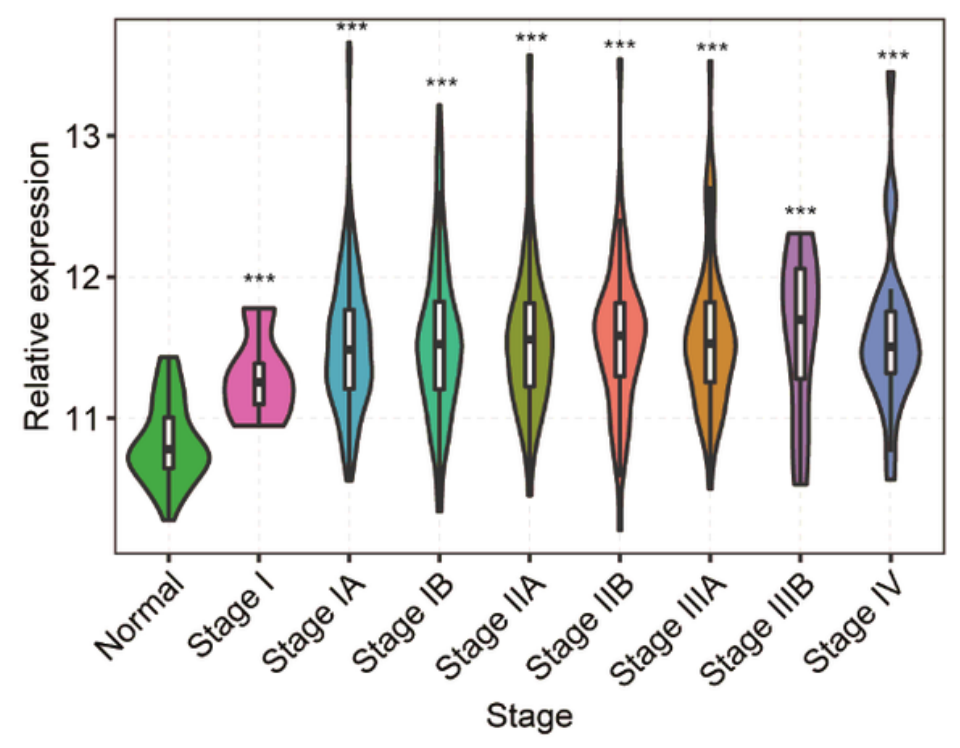

Figure 1

APEX1 expression in NSCLC samples based on the TCGA database. (A) Column chart of APEX1 expression in 517 NSCLC samples and 59 normal samples. APEX1 was significantly higher in lung cancer tissues compared with normal tissue samples. (B) APEX1 distribution of expression levels during different stages of NSCLC samples. NSCLC was staged based on the standards of the International Association for Lung Cancer Research (IASLC) stage (Log-rank test, $p<0.05$ ). 
A

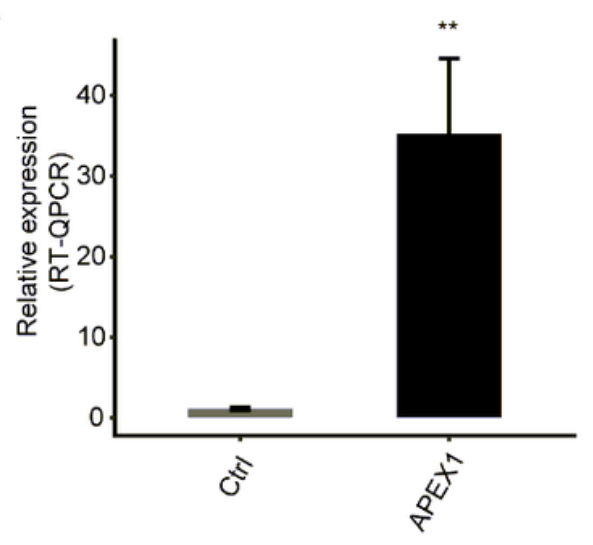

D

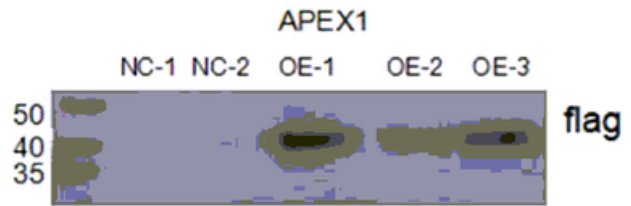

B

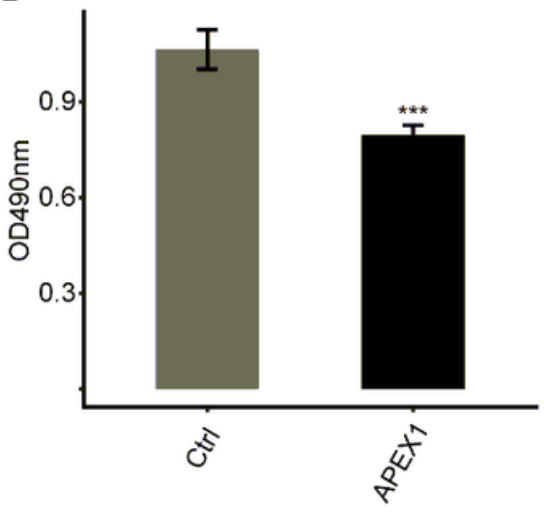

E

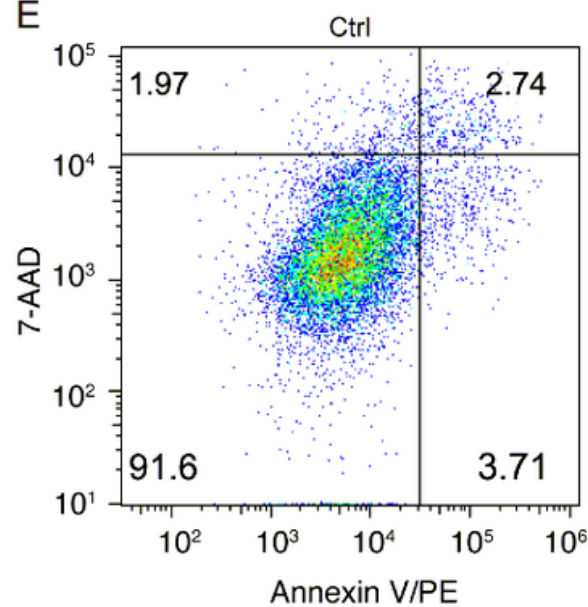

C
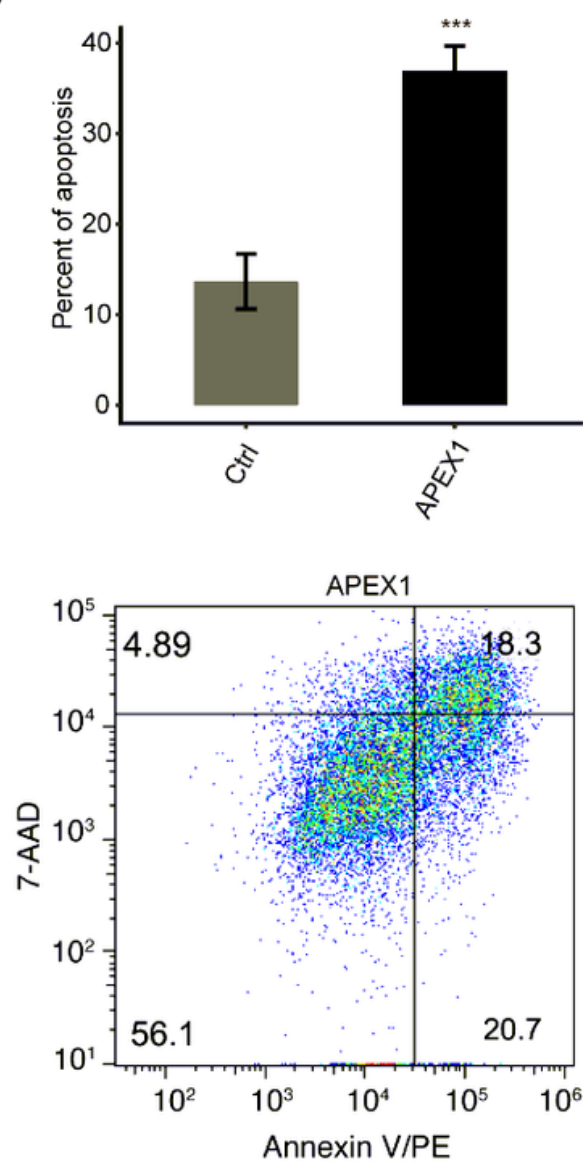

Figure 2

Effects of APEX1 on the proliferation and apoptosis of A549 cells. (A) Expression levels of APEX1 in A549 cells after transient transfection with APEX1 overexpression or control vectors, detected by RTqPCR. (B) Cell proliferation analysis. Proliferation of control and treated A549 cells was assessed with a Luminometer after adding Cell Titer Glo reagent. (C) Apoptosis percentage of A549 cells transfected with APEX1 was quantified. Apoptosis was increased in cells with APEX1 over expression compared to control A549 cells. (D) Western blot analysis showed high levels of APEX1 in cells transfected with the over expression vector vs. the control vector. (E) APEX1 overexpression induced A549 cell apoptosis. APEX1 over expression or control vectors were transfected into A549 cells. The cells were stained with Annexin V/FITC and PI with apoptotic cells detected by flow cytometry. APEX1 OE, expression A549 cells. FITC, fluorescein isothiocyanate; $\mathrm{PI}$, propidium iodide, ${ }^{\star} \mathrm{p}<0.05$. 
A

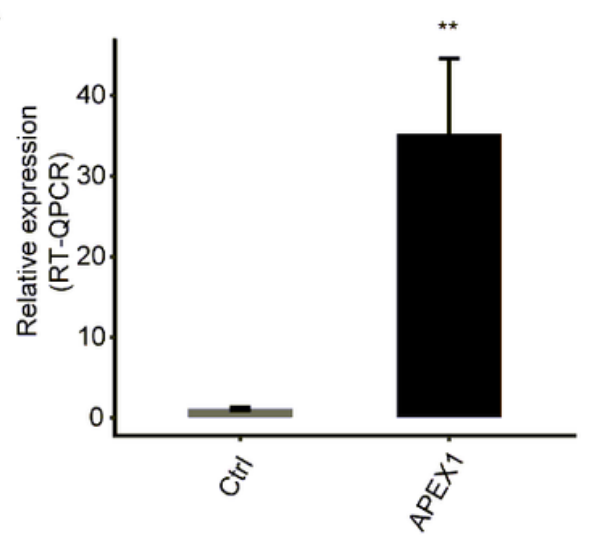

D

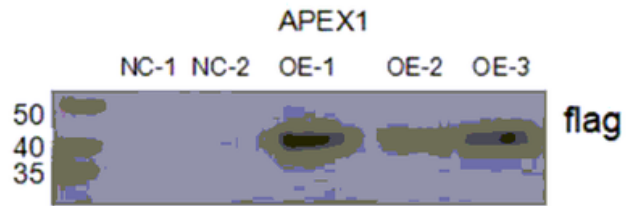

B

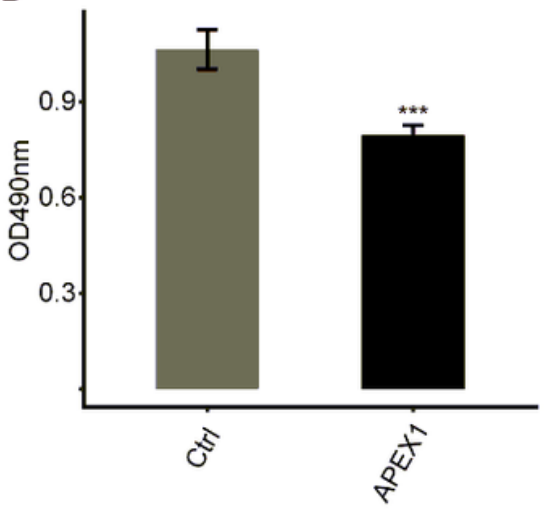

E

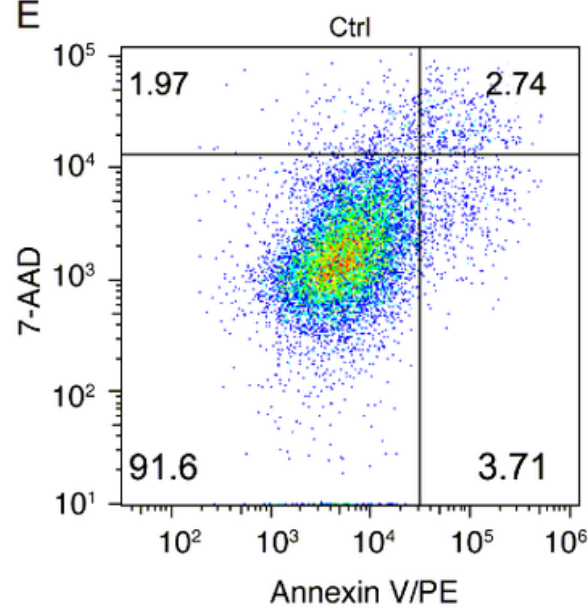

C
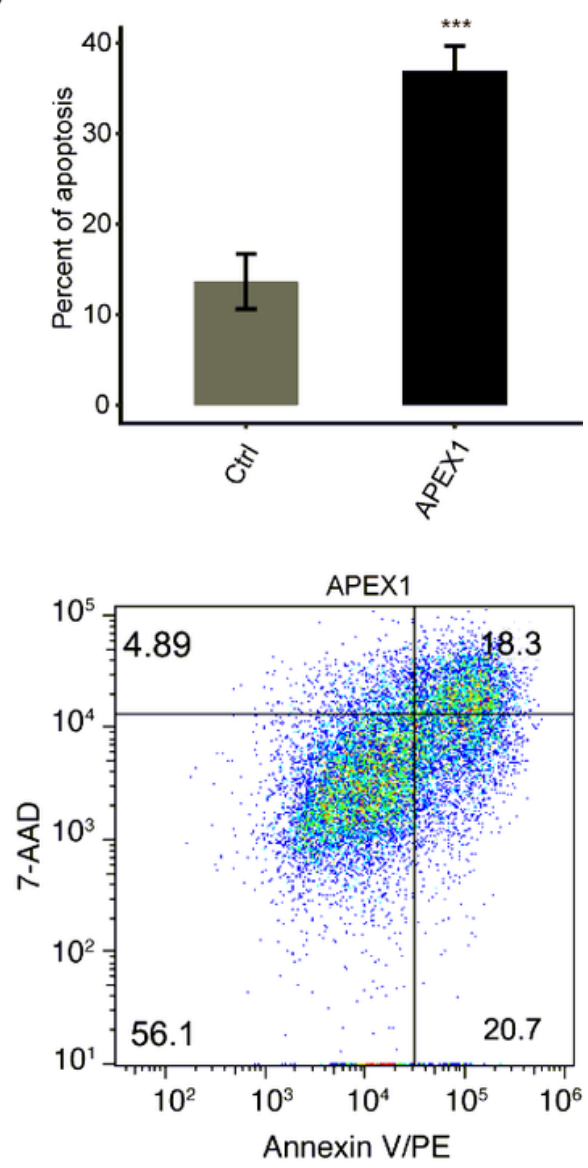

Figure 2

Effects of APEX1 on the proliferation and apoptosis of A549 cells. (A) Expression levels of APEX1 in A549 cells after transient transfection with APEX1 overexpression or control vectors, detected by RTqPCR. (B) Cell proliferation analysis. Proliferation of control and treated A549 cells was assessed with a Luminometer after adding Cell Titer Glo reagent. (C) Apoptosis percentage of A549 cells transfected with APEX1 was quantified. Apoptosis was increased in cells with APEX1 over expression compared to control A549 cells. (D) Western blot analysis showed high levels of APEX1 in cells transfected with the over expression vector vs. the control vector. (E) APEX1 overexpression induced A549 cell apoptosis. APEX1 over expression or control vectors were transfected into A549 cells. The cells were stained with Annexin V/FITC and PI with apoptotic cells detected by flow cytometry. APEX1 OE, expression A549 cells. FITC, fluorescein isothiocyanate; $\mathrm{PI}$, propidium iodide, ${ }^{\star} \mathrm{p}<0.05$. 
A

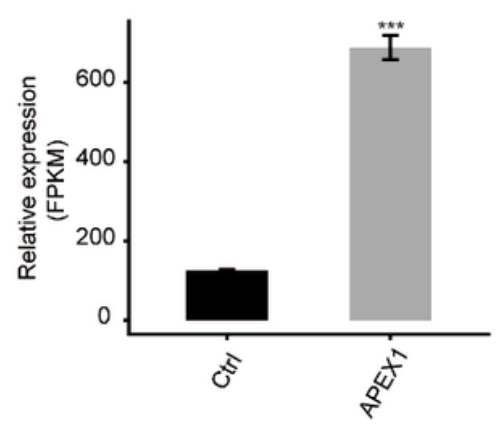

B

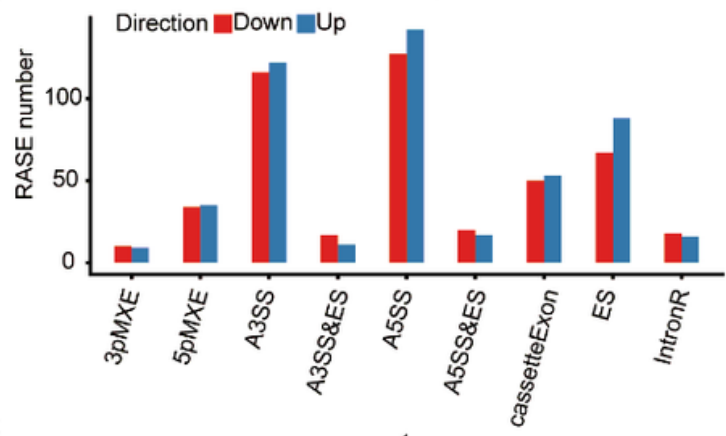

C

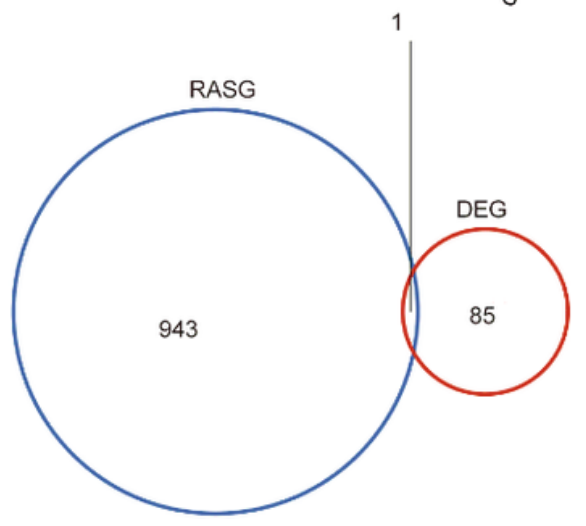

D

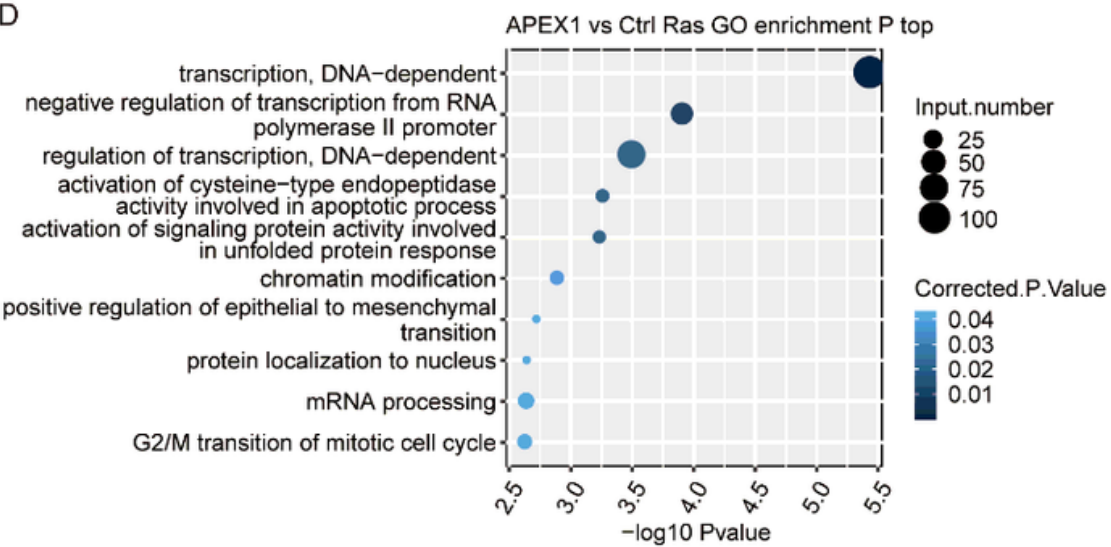

E

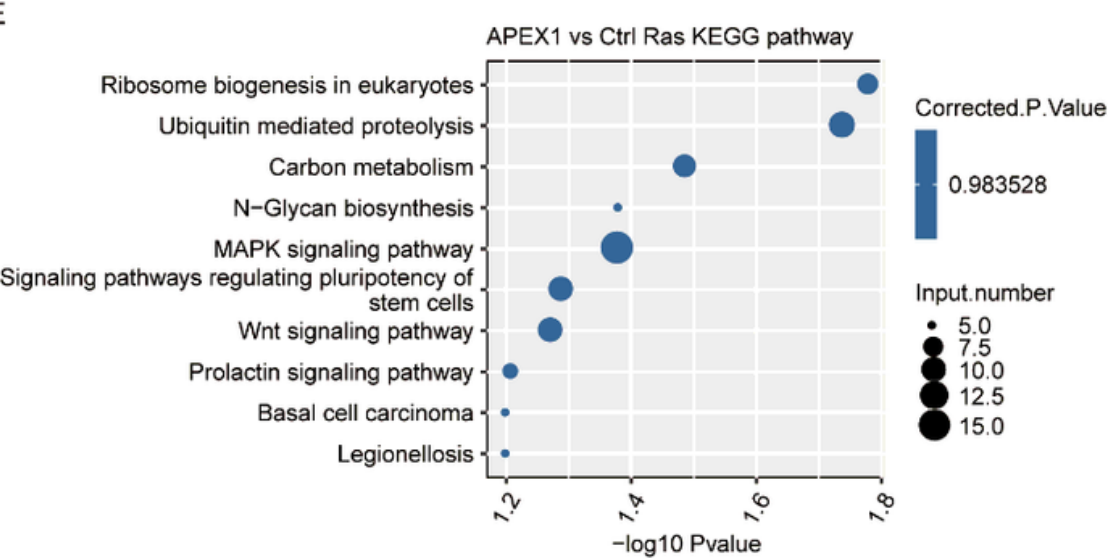

Figure 3

Identification and functional analysis of APEX1-regulated splicing events in A549 cells. (A) APEX1 expression quantified by RNA sequencing and qRT-PCR. FPKM values were calculated as described in Materials and Methods. (B) Classification of different AS types regulated by APEX1 protein. (C) Overlap analysis between APEX1-regulated differentially expressed genes (DEG) and alternative splicing genes (RASG). (D) The top $10 \mathrm{GO}$ biological processes. (E) KEGG functional pathway analysis of AS genes. Node size represents the number of genes in the pathways. 
A

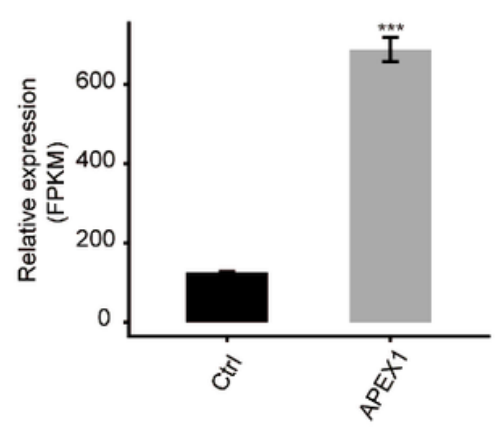

B

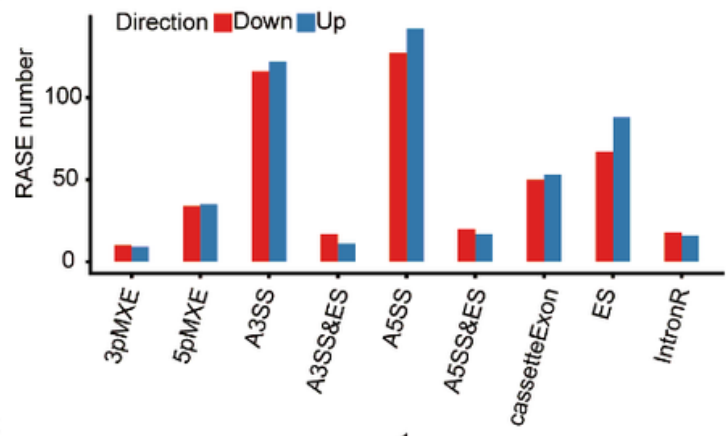

C

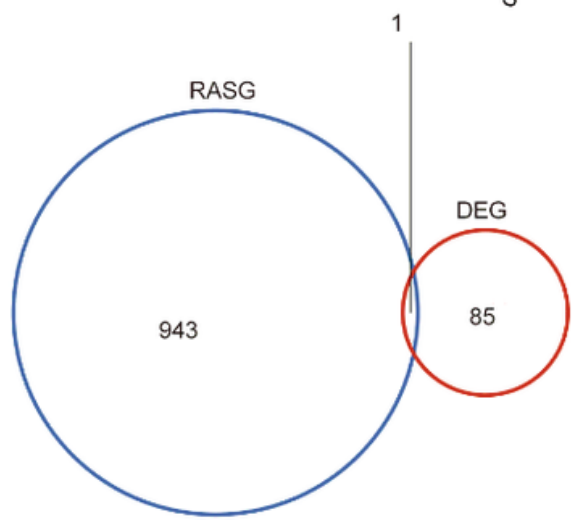

D

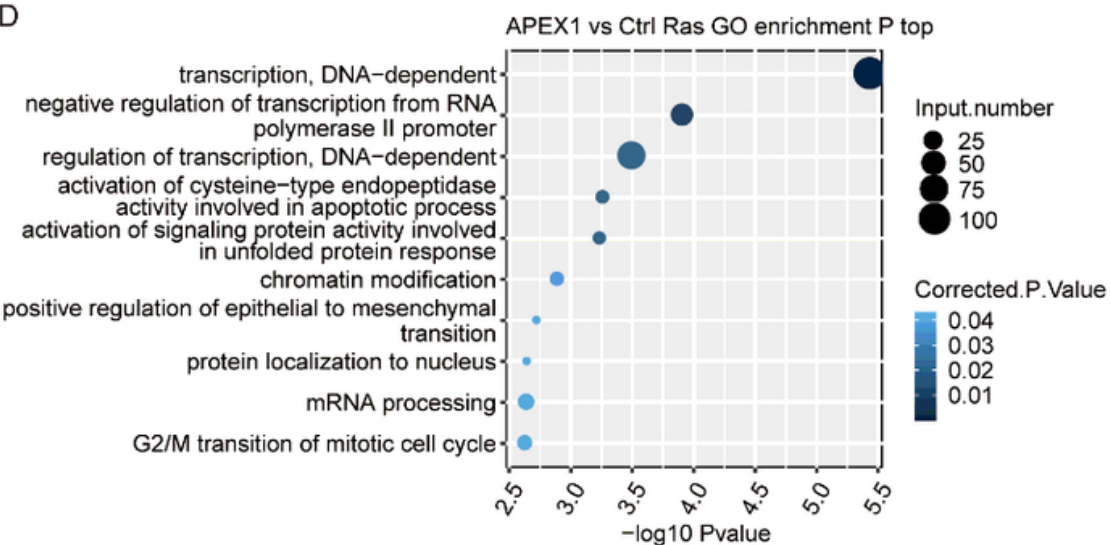

E

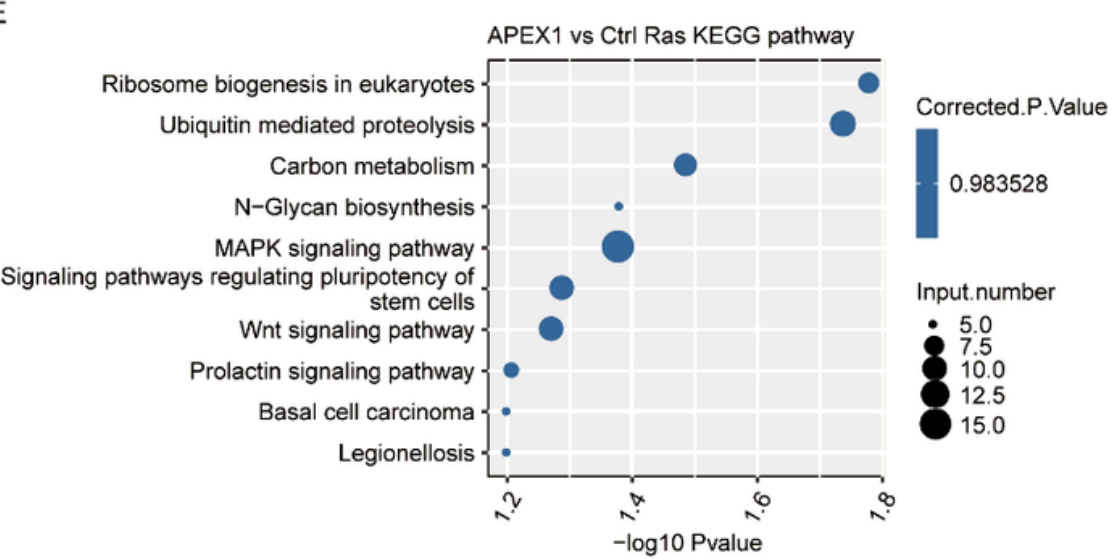

Figure 3

Identification and functional analysis of APEX1-regulated splicing events in A549 cells. (A) APEX1 expression quantified by RNA sequencing and qRT-PCR. FPKM values were calculated as described in Materials and Methods. (B) Classification of different AS types regulated by APEX1 protein. (C) Overlap analysis between APEX1-regulated differentially expressed genes (DEG) and alternative splicing genes (RASG). (D) The top $10 \mathrm{GO}$ biological processes. (E) KEGG functional pathway analysis of AS genes. Node size represents the number of genes in the pathways. 

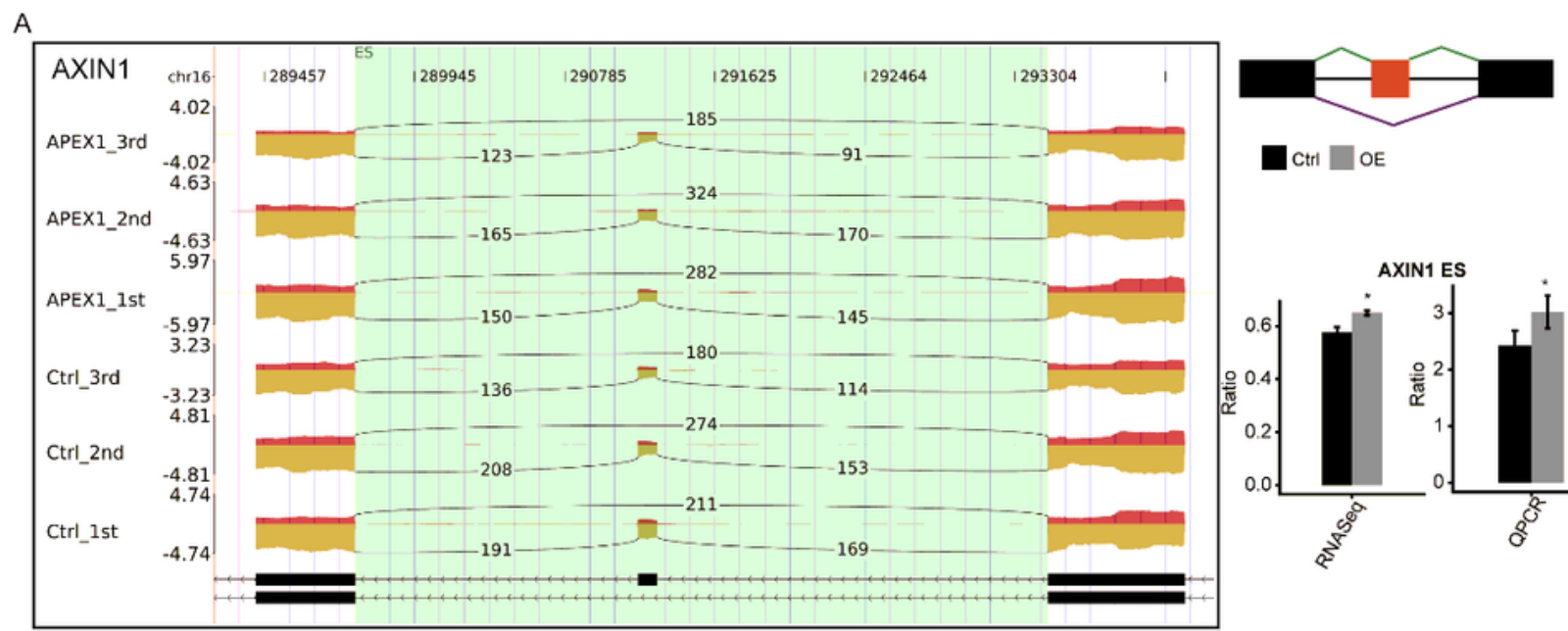

Ctrl
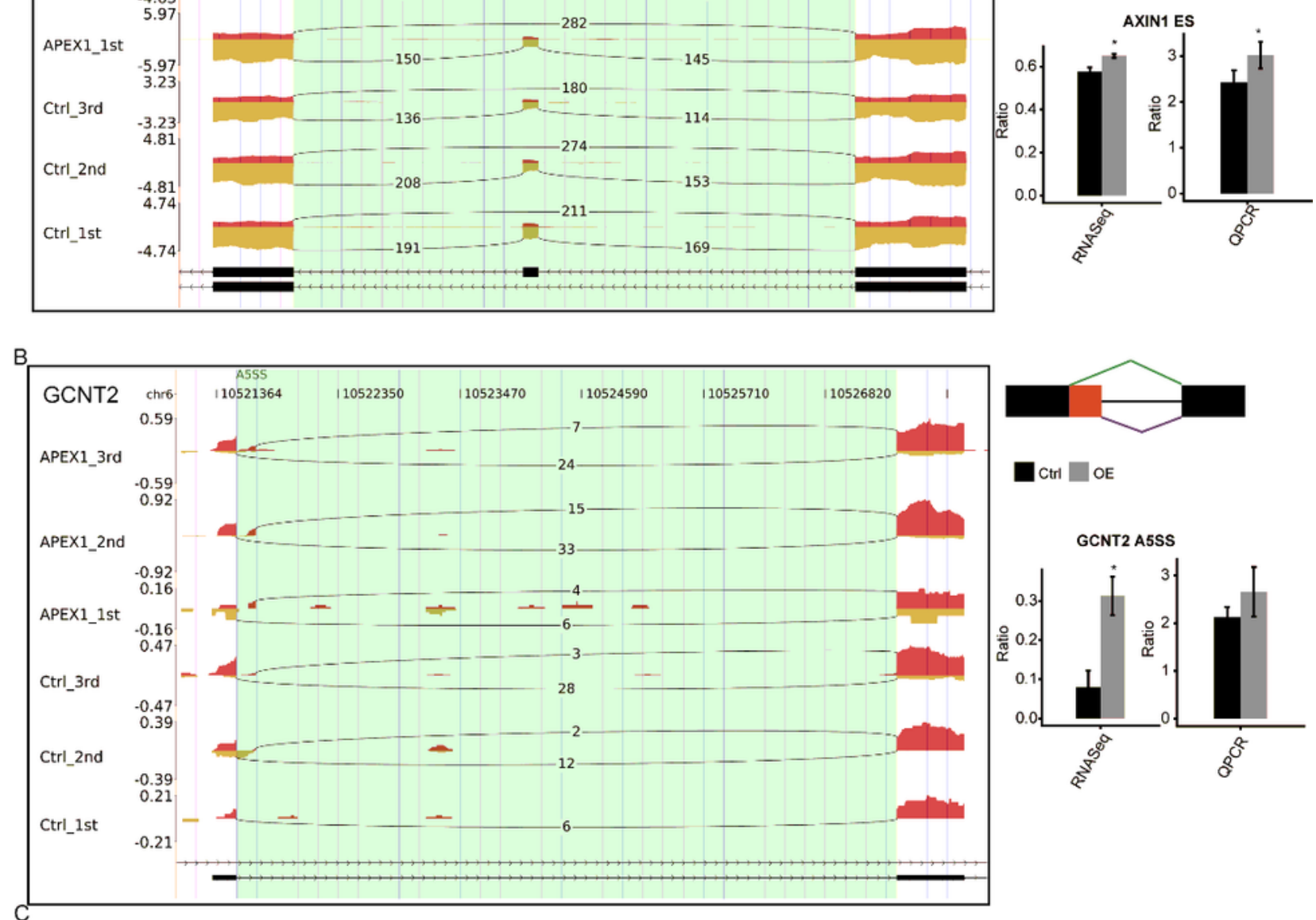

口
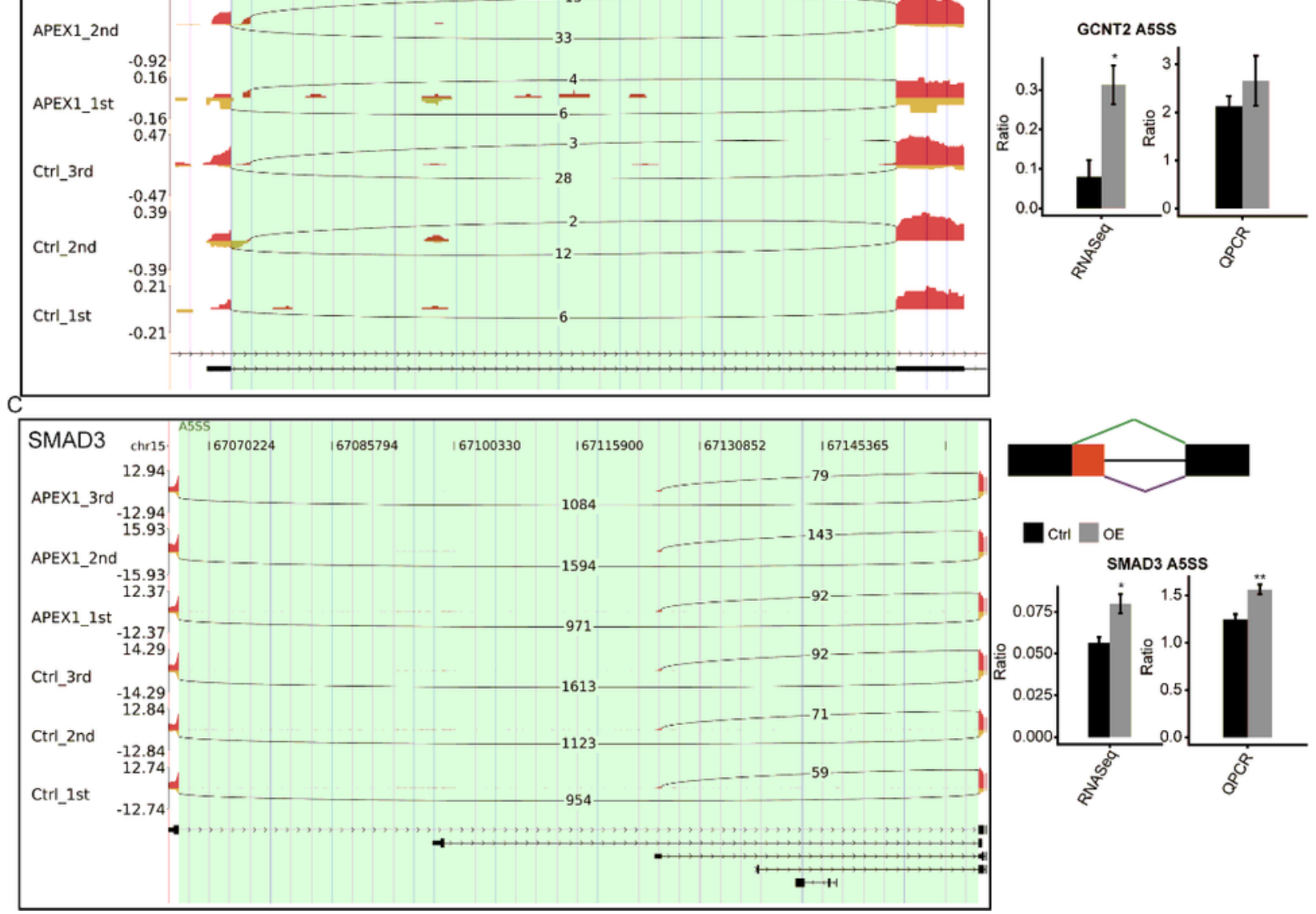

Ctrl

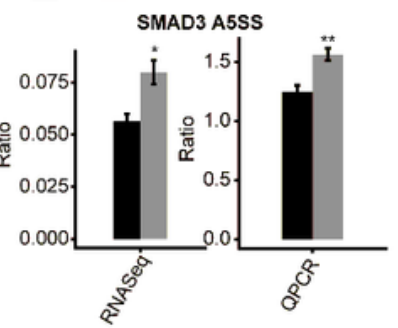

\section{Figure 4}

Validation of APEX1-RAES. (Left panel) IGV-sashimi plots showed one ES (A) and two A5SS (B, C) alternative splicing events in three different genes. Read distribution of each alternative splicing event was plotted in the left panel with the transcripts of each gene shown below. The schematic diagrams depict the structures of ASEs, AS1 (purple line) and AS2 (green line). The exon sequences are denoted by boxes and intron sequences by horizontal lines (right panel, top). RNA-seq quantification and RT-qPCR 
validation of ASEs are shown at the bottom of the right panel. The altered ratios of AS events in RNA-seq were calculated using the formula: AS1 junction reads/AS1junction reads +AS2 junction reads; while the altered ratio of AS events in q-PCR were calculated using the formula: AS1 transcripts level/AS2 transcripts level. (For interpretation of the references to color in this figure legend, the reader is referred to the web version of this article.)
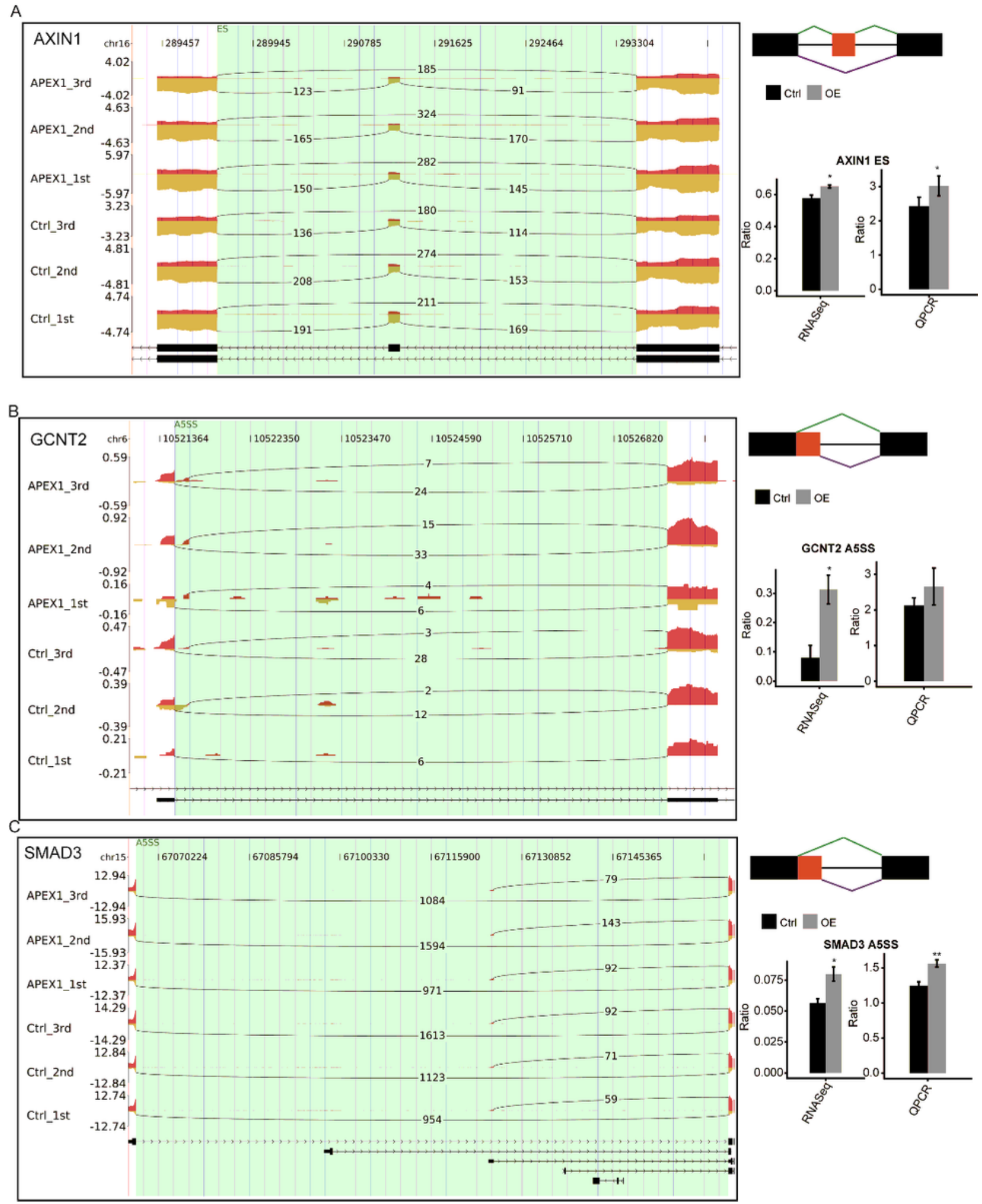

\section{Figure 4}


Validation of APEX1-RAES. (Left panel) IGV-sashimi plots showed one ES (A) and two A5SS (B, C) alternative splicing events in three different genes. Read distribution of each alternative splicing event was plotted in the left panel with the transcripts of each gene shown below. The schematic diagrams depict the structures of ASEs, AS1 (purple line) and AS2 (green line). The exon sequences are denoted by boxes and intron sequences by horizontal lines (right panel, top). RNA-seq quantification and RT-qPCR validation of ASEs are shown at the bottom of the right panel. The altered ratios of AS events in RNA-seq were calculated using the formula: AS1 junction reads/AS1junction reads +AS2 junction reads; while the altered ratio of AS events in q-PCR were calculated using the formula: AS1 transcripts level/AS2 transcripts level. (For interpretation of the references to color in this figure legend, the reader is referred to the web version of this article.)

A
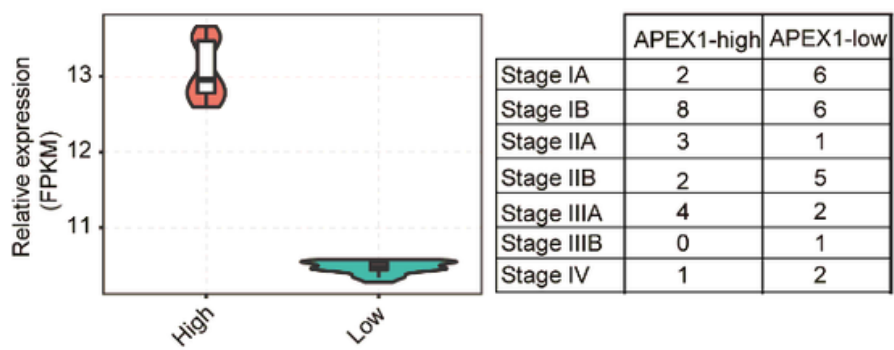

B

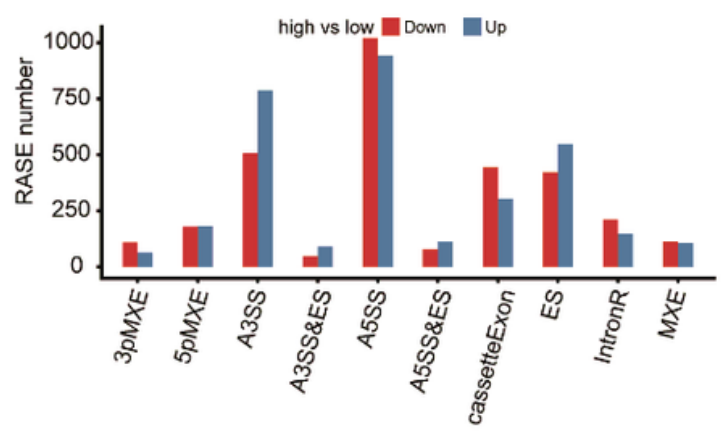

C

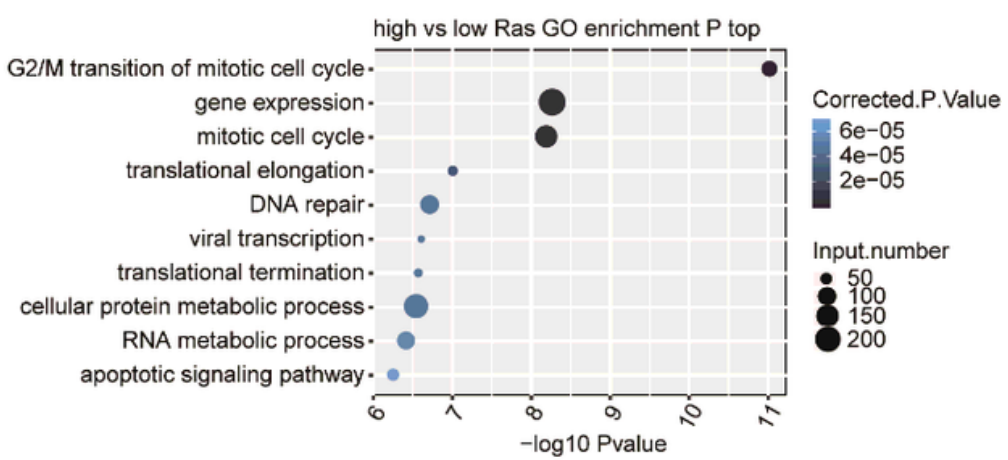

D

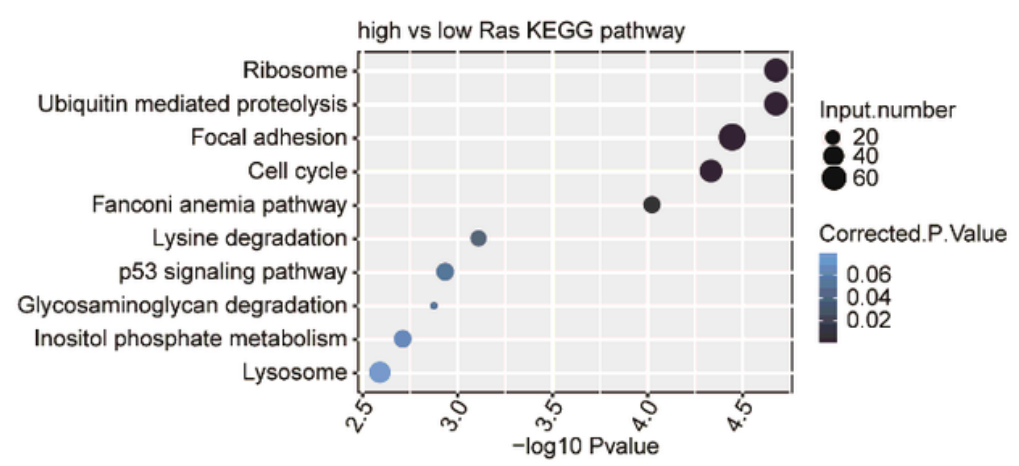

\section{Figure 5}

Analysis of potential APEX1-RASEs and genes in NSCLC clinical samples. (A) Violin plot of APEX1 expression in two groups of samples with APEX1 differentially expressed. The samples were grouped based on their APEX1 expression levels regardless of their pathological stage. (B) Bar plot of the distribution of AS events showing significant difference between the high- and low-APEX1 samples. The regulated AS events (RASE) were classified into 10 different types as detailed in Materials and Methods. The red and blue bars indicate the number of AS events upregulated and downregulated, respectively, in the high-APEX1 groups compared to the low-APEX1 group. (C) The top ten GO biological processes. (D) KEGG functional pathway of RASE genes regulated by APEX1. Functional clustering of RASE genes regulated by APEX1 within the TCGA data. 
A

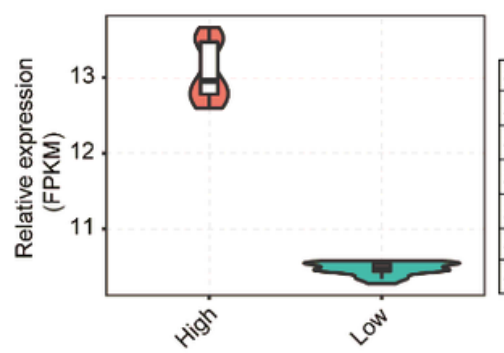

\begin{tabular}{|l|c|c|}
\cline { 2 - 3 } \multicolumn{1}{c|}{} & APEX1-high & APEX1-low \\
\hline Stage IA & 2 & 6 \\
\hline Stage IB & 8 & 6 \\
\hline Stage IIA & 3 & 1 \\
\hline Stage IIB & 2 & 5 \\
\hline Stage IIIA & 4 & 2 \\
\hline Stage IIIB & 0 & 1 \\
\hline Stage IV & 1 & 2 \\
\hline
\end{tabular}

B

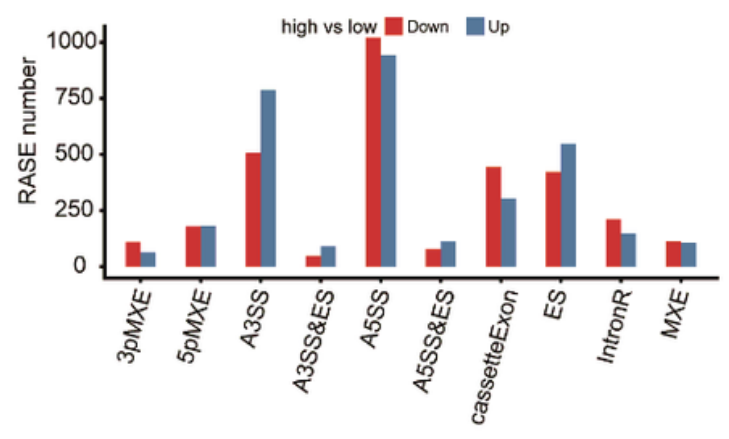

C

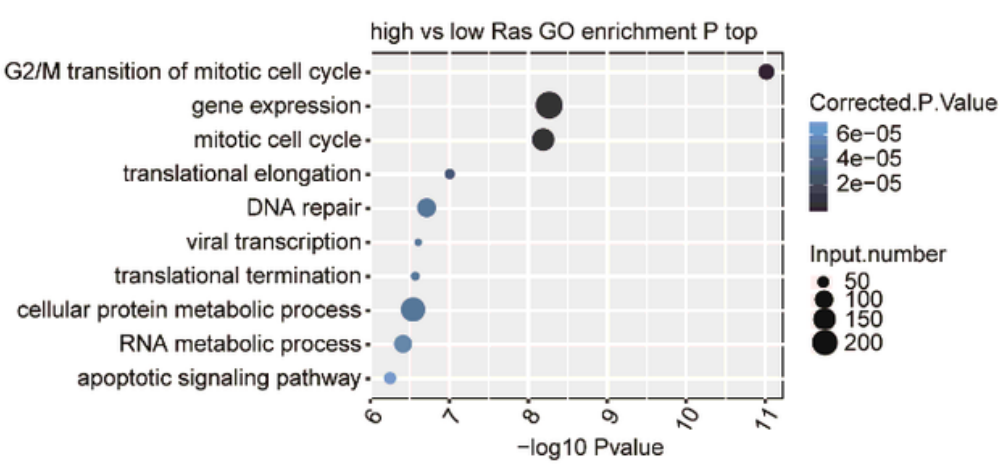

D

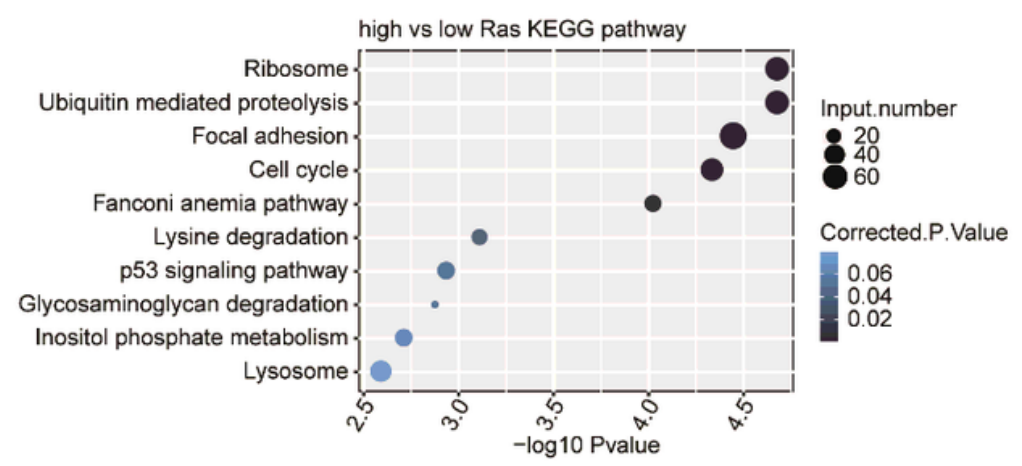

\section{Figure 5}

Analysis of potential APEX1-RASEs and genes in NSCLC clinical samples. (A) Violin plot of APEX1 expression in two groups of samples with APEX1 differentially expressed. The samples were grouped based on their APEX1 expression levels regardless of their pathological stage. (B) Bar plot of the distribution of AS events showing significant difference between the high- and low-APEX1 samples. The regulated AS events (RASE) were classified into 10 different types as detailed in Materials and Methods. The red and blue bars indicate the number of AS events upregulated and downregulated, respectively, in the high-APEX1 groups compared to the low-APEX1 group. (C) The top ten GO biological processes. (D) KEGG functional pathway of RASE genes regulated by APEX1. Functional clustering of RASE genes regulated by APEX1 within the TCGA data. 


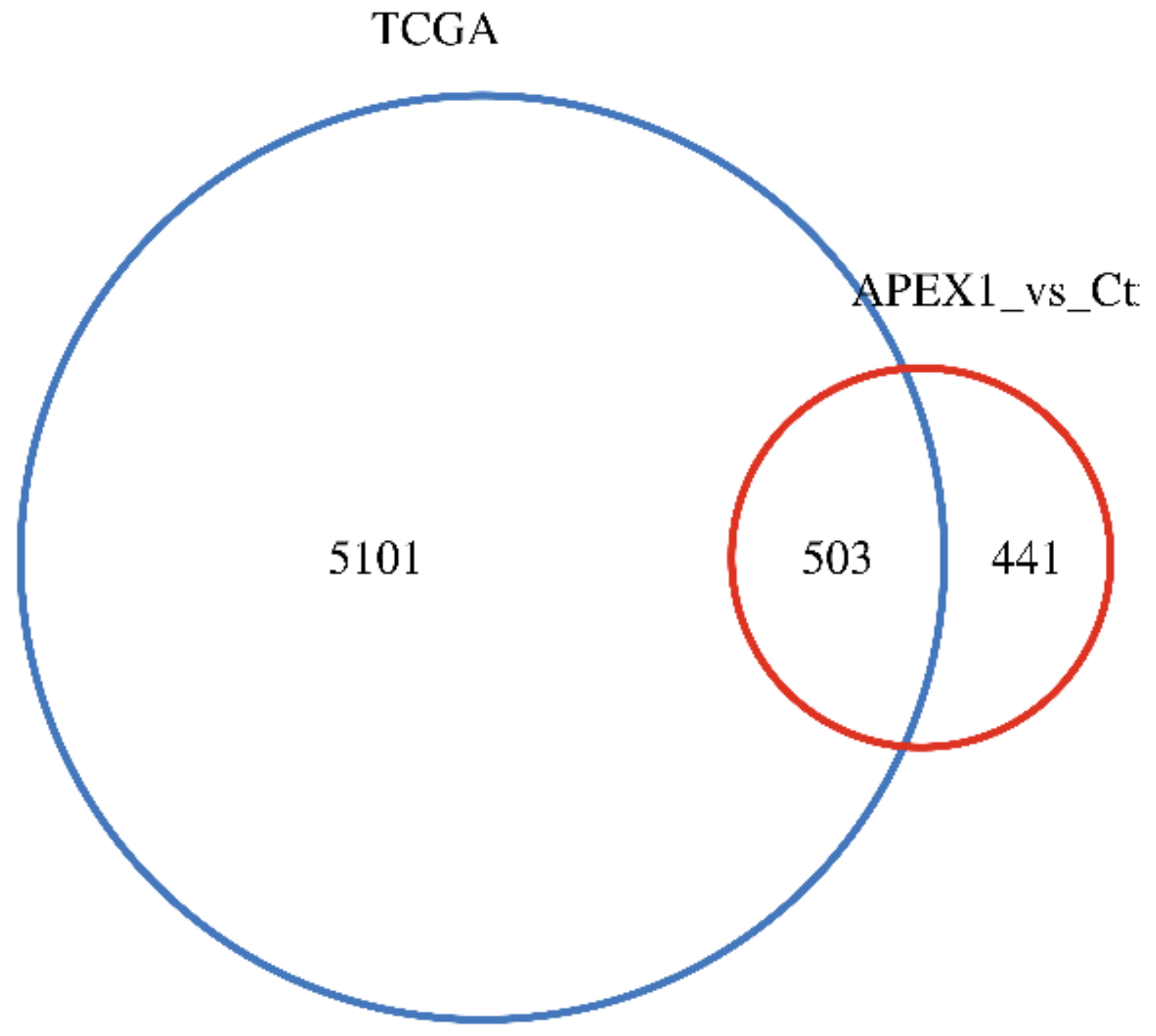

Figure 6

Venn diagram showing overlapped RASE genes due to APEX1-regulation in the TCGA data set for NSCLC cancer and A549 cells, $p<0.05$. 


\section{TCGA}

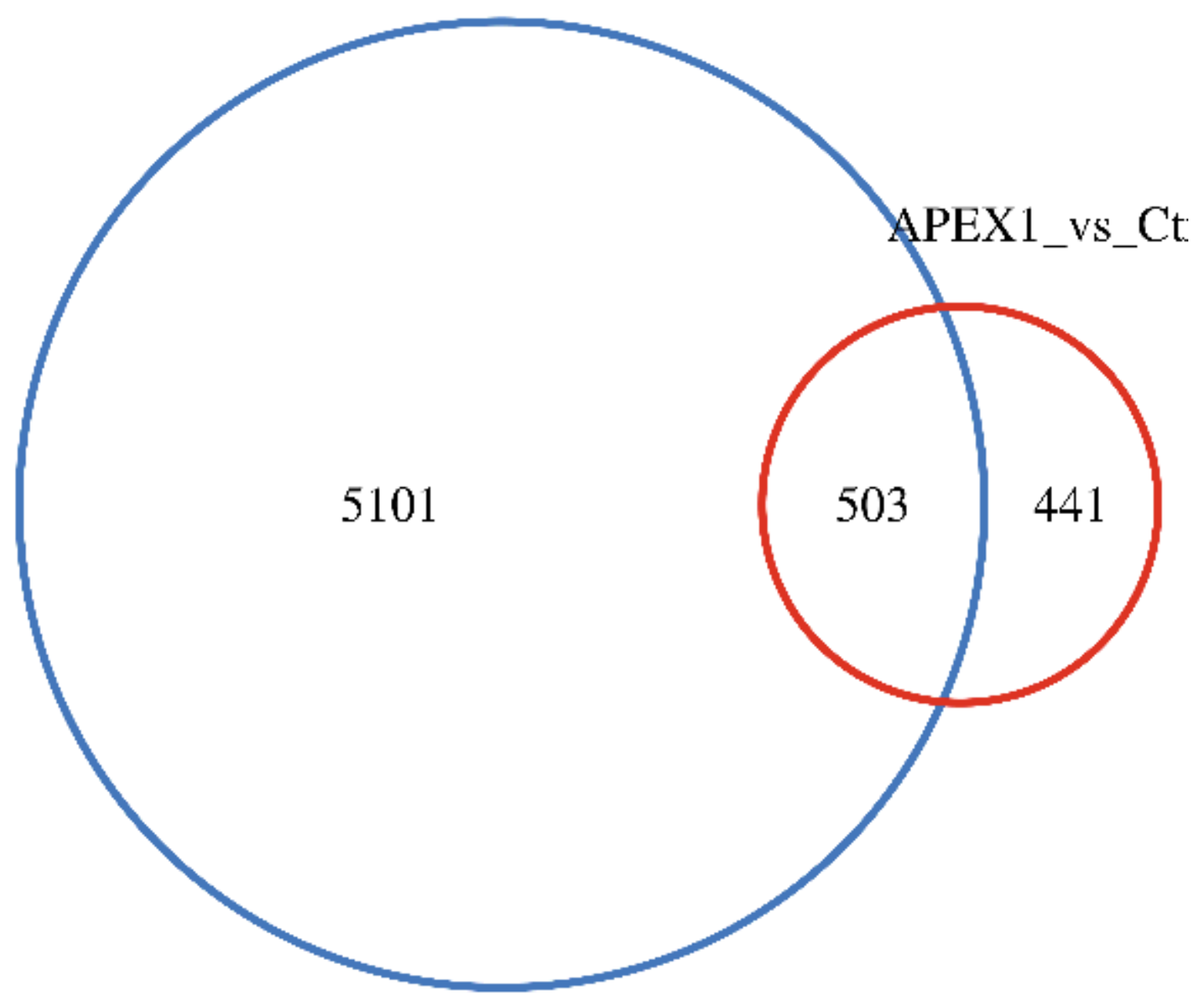

Figure 6

Venn diagram showing overlapped RASE genes due to APEX1-regulation in the TCGA data set for NSCLC cancer and A549 cells, $p<0.05$.

\section{Supplementary Files}

This is a list of supplementary files associated with this preprint. Click to download.

- Additionalfile1TheprimersfordetectingASEs.xlsx

- Additionalfile1TheprimersfordetectingASEs.xlsx

- Additionalfile2HiSeqV2APEX1expressionbox.xlsx

- Additionalfile2HiSeqV2APEX1expressionbox.xIsx

- Additionalfile3Mappingofcleanreads.xlsx 
- Additionalfile3Mappingofcleanreads.xlsx

- Additionalfile4AllknownASeventsdetectedbytype.xlsx

- Additionalfile4AllknownASeventsdetectedbytype.xlsx

- Additionalfile5ClassificationofallRASEevents.xIsx

- Additionalfile5ClassificationofallRASEevents.xIsx

- Additionalfile6RasKEGGpathwayidentifyOriginal.xIsx

- Additionalfile6RasKEGGpathwayidentifyOriginal.xIsx

- Additionalfile7ValidationofAPEX1RASE.tif

- Additionalfile7ValidationofAPEX1RASE.tif

- Additionalfile8Mappingofcleanreads.xIsx

- Additionalfile8Mappingofcleanreads.xlsx 